\title{
Technè
}

La science au service de l'histoire de l'art et de la préservation des biens culturels

45 | 2017

Bronzes grecs et romains : études récentes sur la statuaire antique

\section{Dorure et redorure de la statuaire antique en bronze : une nouvelle lecture de l'Apollon de Lillebonne}

Gilding and regilding of ancient bronze statuary: new observations on the Apollo from Lillebonne

Dominique Robcis, Sophie Descamps-Lequime, Nathalie Pingaud et Benoît Mille

\section{OpenEdition Journals}

Édition électronique

URL : http://journals.openedition.org/techne/1324

DOI : 10.4000/techne.1324

ISSN : 2534-5168

Éditeur

C2RMF

\section{Édition imprimée}

Date de publication : 1 mai 2017

Pagination : 100-113

ISBN : 978-2-7118-6408-9

ISSN : $1254-7867$

Référence électronique

Dominique Robcis, Sophie Descamps-Lequime, Nathalie Pingaud et Benoît Mille, « Dorure et redorure de la statuaire antique en bronze : une nouvelle lecture de l'Apollon de Lillebonne », Technè [En ligne], 45 | 2017, mis en ligne le 19 décembre 2019, consulté le 23 juillet 2020. URL : http:// journals.openedition.org/techne/1324 ; DOI : https://doi.org/10.4000/techne.1324

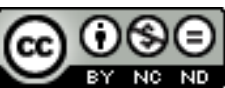

La revue Technè. La science au service de l'histoire de l'art et de la préservation des biens culturels est mise à disposition selon les termes de la Licence Creative Commons Attribution - Pas d'Utilisation Commerciale - Pas de Modification 4.0 International. 


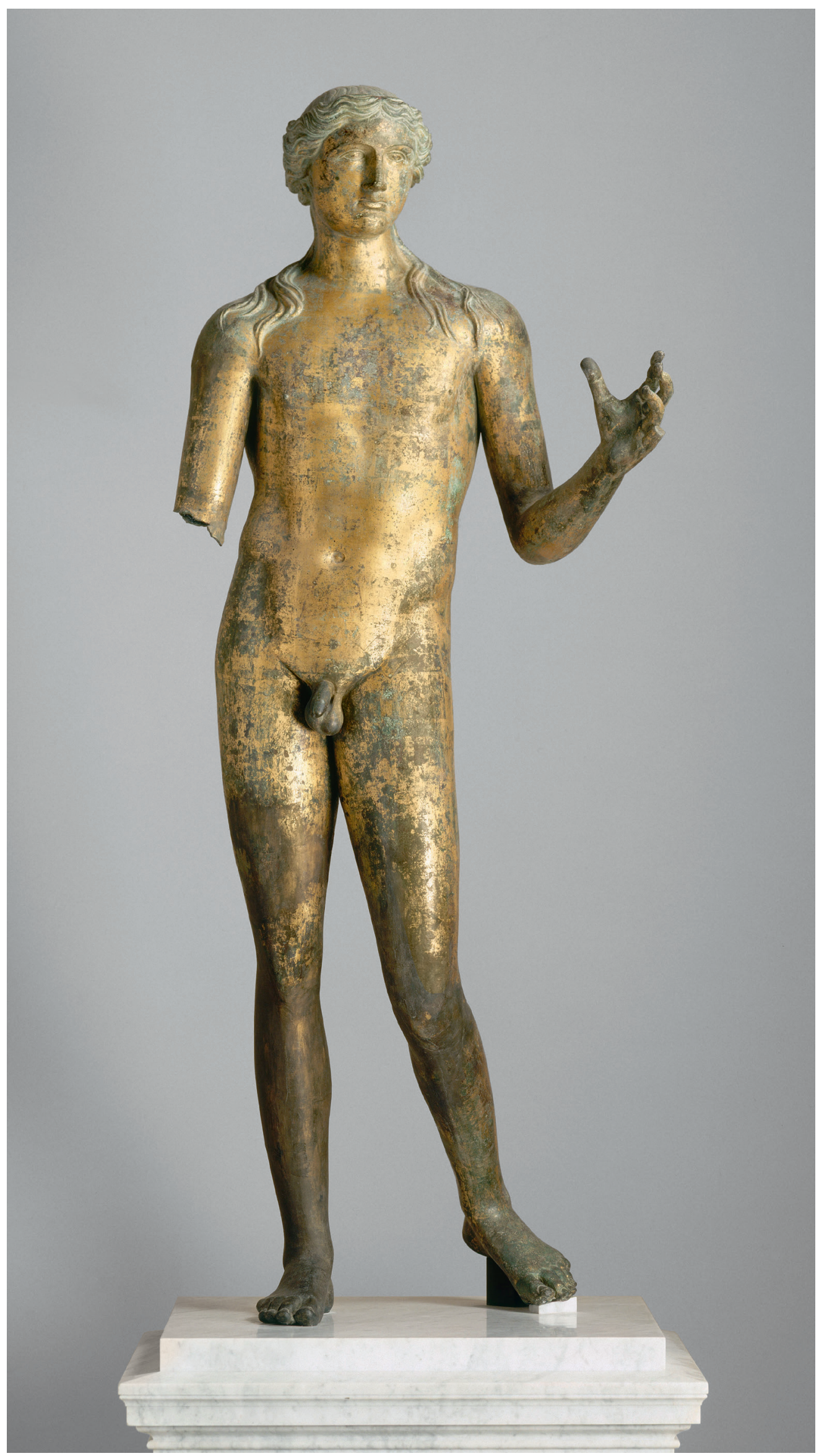

Fig. 1. L'Apollon de Lillebonne, Paris, musée du Louvre, département des Antiquités grecques, étrusques et romaines, bronze doré, H. 1,94 m. ๑ RMN-

Grand Palais (musée du Louvre)/Hervé Lewandowski. 
Dominique Robcis

Sophie Descamps-Lequime

Nathalie Pingaud

Benoît Mille

\section{Dorure et redorure de la statuaire antique en bronze : une nouvelle lecture de l'Apollon de Lillebonne}

Gilding and regilding of ancient bronze statuary: new observations on the Apollo from Lillebonne

Résumé. L'Apollon de Lillebonne est, à ce jour, la plus grande sculpture de bronze représentant une divinité, découverte en Gaule romaine. Datée stylistiquement du II ${ }^{e}$ siècle de notre ère, la statue, qui figure un Apollon lyricine, provient de l'antique Juliobona (Lillebonne, Normandie). Divinité salutaire, il s'agit très vraisemblablement d'une statue de culte. Une première étude de laboratoire avait été menée lors d'une campagne de restauration en 1974-1975 sous la direction d'Albert France-Lanord. Une exploration scientifique complémentaire, conduite au C2RMF en 2015, a apporté des informations inédites sur le contexte de présentation de l'Apollon dans l'Antiquité. Elle a permis surtout de caractériser la technique de dorure du bronze et aussi de mettre en évidence une importante phase de redorure antique. L'étude de la dorure a fait appel à diverses techniques d'examens et d'analyses : métallographie, microscopie 3D, spectrométrie de fluorescence X, microscopie électronique à balayage et à effet de champ.

Mots-clés. Apollon, Lillebonne, grande statuaire, bronze, dorure à la feuille, redorure, réparure, métallographie, restauration, microscopie numérique 3D, polissage ionique.
Abstract. The Lillebonne Apollo is the largest bronze sculpture of a divinity found so far in Roman Gaul. Stylistically dating from the 2 nd century AD, this statue of Apollo holding a lyre (now lost) was discovered in ancient Juliobona (present-day Lillebonne, Normandy, France). Given that Apollo was considered a salvation deity, this was very probably a cult statue. An initial laboratory study was conducted during the restoration of the sculpture in 1974-1975 under the direction of Albert France-Lanord. A complementary scientific investigation, carried out at the C2RMF in 2015, revealed new data about the context in which the Apollo was displayed in Antiquity. In particular, it identified the gilding technique used on the bronze and brought to light an important stage of ancient regilding. The study of the gilding involved diverse testing and analytical techniques: metallography, $3 D$ digital microscopy, X-ray fluorescence, scanning electron microscope.

Keywords. Apollo, Lillebonne, large statuary, bronze, gold leaf gilding, regilding, fettling, metallography, restoration, $3 D$ digital microscopy, ion-beam polishing.

\section{Découverte de l'Apollon et premières interrogations}

La trouvaille fortuite de l'Apollon (fig. 1) ${ }^{1}$, le 23 juillet 1823, par des ouvriers qui travaillaient sur un terrain appartenant à Guillaume Léonard Holley ${ }^{2}$, à quelque trois cents mètres à l'est des vestiges du théâtre romain de Lillebonne (l'antique Juliobona) et «à trois pieds seulement de la surface du champ ${ }^{3}$ ", devait immédiatement susciter l'intérêt de la communauté scientifique, tant locale que parisienne et anglaise. Bien que lacunaire, l'œuvre frappa d'emblée par ses dimensions imposantes et par la dorure qui recouvrait son épiderme, au point que l'on crut que les fragments manquants avaient été fondus secrètement pour récupérer le métal précieux. Il n'en était rien, mais plusieurs morceaux isolés furent distribués et certains sacrifiés pour préciser la nature de la dorure et de l'alliage ${ }^{4}$. Dès la découverte de la statue, le fouilleur du théâtre, François Rever, avait procédé à un examen, dont il faut saluer la clairvoyance et la précision ${ }^{5}$. Le dessinateur Langlois se rendit sur place à la fin du mois d'août pour restituer l'apparence de la statue ${ }^{6}$ (fig. 2), et d'éminents spécialistes, tel le comte de Clarac, conservateur des antiques du Musée royal, s'arrêtèrent à Lillebonne : «En passant hier à Lillebonne j'ai vu avec grand plaisir une statue antique qui y a été découverte il y a deux mois [...] Cette statue est de grande proportion [...] La dorure en est superbe, très épaisse, ce qu'il est facile de voir sur la tranche des fragments. Elle est presque partout très bien conservée, et je ne doute pas qu'elle ne reparaisse avec tout son éclat, lorsqu'on aura enlevé le tartre et la croute (sic) qui recouvre certaines parties [...] les statues de bronze dorées de grandes dimensions sont de la plus grande rareté. Il me semble que l'on ne connaît de ce genre que les chevaux de Venise et encore la dorure n'existe-t-elle plus $[\ldots]^{7}$. » Alphonse de Cailleux,

Dominique Robcis, chef de travaux d'art, C2RMF (dominique.robcis@culture.gouv.fr). Sophie Descamps-Lequime, conservateur général du patrimoine, musée du Louvre, département des Antiquités grecques, étrusques et romaines (sophie.descamps@louvre.fr). Nathalie Pingaud, assistante ingénieure, C2RMF (nathalie.pingaud@culture.gouv.fr). Benoît Mille, ingénieur d'étude, C2RMF et Préhistoire et Technologie, UMR 7055, Nanterre (benoit.mille@ culture.gouv.fr). 


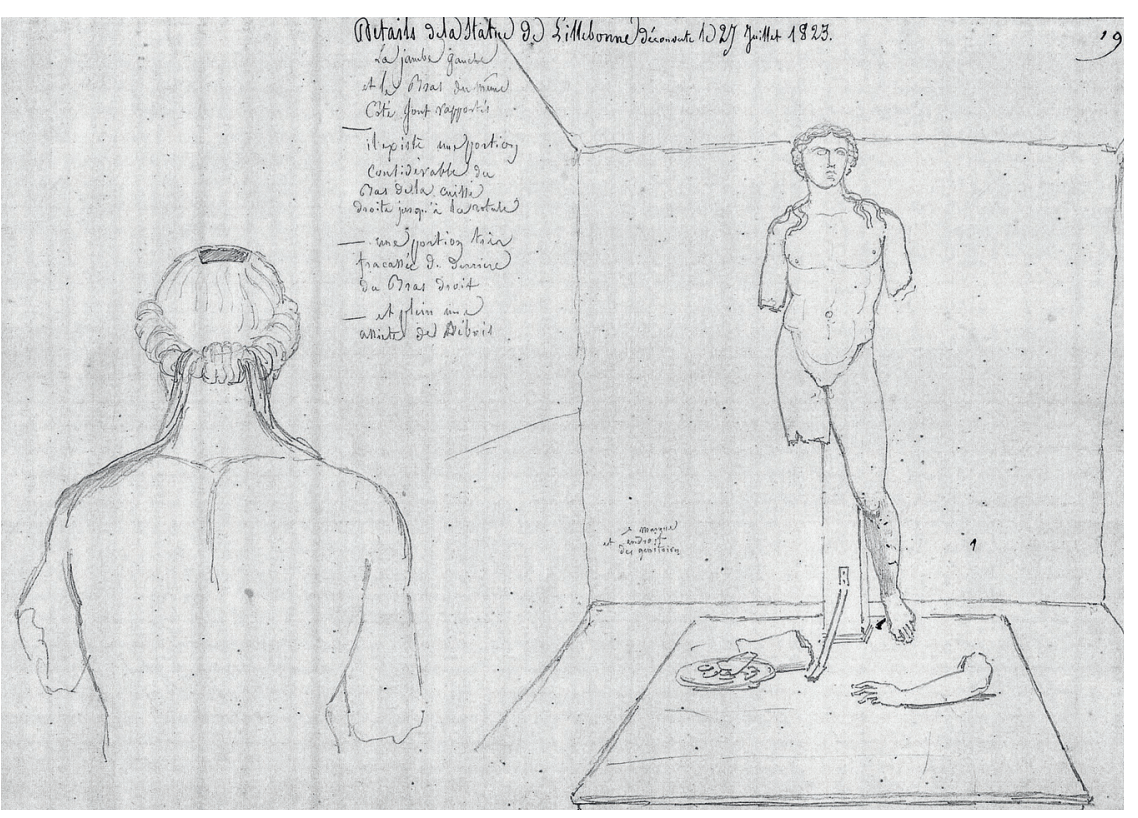

Fig. 2. Dessin de la statue, réalisé en août 1823 par Eugène Hyacinthe Langlois, album de la Commission départementale des Antiquités de Seine-Maritime, Archives départementales de Seine-Maritime, 6 Fi 3 p. 22 (détail). (C) J.-P. Godais, Musée d'Art, Histoire et Archéologie, Ville d'Évreux, 2013.

secrétaire général des musées, fut officiellement autorisé le 29 octobre 1823 «à se transporter sur les lieux pour y examiner la statue $[\ldots]^{8}$ ». Il fallait juger de l'importance de l'œuvre et estimer sa valeur. Holley souhaitait en effet céder le bronze au musée du Louvre, mais ses prétentions exorbitantes se heurtèrent rapidement à la réticence des autorités et aux jugements mitigés de certains. La statue ne correspondait pas aux critères qui fondaient alors l'histoire de l'art de l'Antiquité, de sorte que l'impression générale qui se dégageait de son étude n'était pas nécessairement favorable. C'est ce dont le sculpteur François-Joseph Bosio se fait l'écho dans une lettre datée du 5 janvier 1824 : "C'est un antique de la $4^{\mathrm{e}}$ classe, qui n'est ni bon, ni mauvais. La tête ne vaut pas grand-chose, la main gauche n'est pas d'ensemble ${ }^{9}$. » Le 31 octobre 1824, le vicomte de la Rochefoucault, chargé du département des Beaux-Arts au ministère de la Maison du roi, s'adressait au comte de Forbin, directeur des Musées royaux, lui demandant de s'adjoindre MM. de Clarac et Bosio afin d'établir un rapport qui devait porter notamment sur l'époque de la statue, sur son attribution à une sphère de production grecque ou romaine et sur la dorure : «La dorure qui la recouvre peutelle être attribuée à l'Antiquité ? Ne doit-on pas la considérer plutôt comme ayant été faite après coup et dans le Moyen Âge, où ce procédé était plus usité que chez les Anciens ${ }^{10}$ ? » La statue était alors à Paris ${ }^{11}$. Elle y avait été mise en gage et fut acquise en 1825 par un Anglais, Samuel Woodburn, qui espérait la proposer au British Museum ${ }^{12}$. Ses espoirs furent déçus, après ceux de Holley, et ni l'un ni l'autre ne connurent de leur vivant le rapatriement de l'Apollon en France en 1853, pour son acquisition par le musée du Louvre. Le dossier était alors suivi par Adrien de Longpérier, nouveau conservateur des antiques du Louvre, parti à Londres négocier l'achat et contrôler les bonnes conditions du retour de l'œuvre à Paris : «Je ne sais si vous serez content de la statue ", écrivait-il le 29 juillet 1853 au comte de Nieuwerkerke, directeur général des Musées impériaux, «elle a la jambe droite au-dessous du genou cassée et perdue. L'avant-bras droit manque également [...] C'est un Apollon à coiffure féminine qui, sans être d'un style admirable, a cependant beaucoup de grandeur. La dorure $\mathrm{du}$ torse est encore assez vive, et il serait facile de la rendre encore plus apparente par un nettoyage. En somme, c'est un morceau extrêmement remarquable qui produira beaucoup d'effet dans le Musée et qu'il eût été très malheureux de laisser échapper, car les bronzes de cette dimension sont tout ce qu'il y a de plus rare ${ }^{13}$. »

\section{Particularités techniques et interventions modernes sur la statue}

\section{Réparure}

L'examen du « tronçon de la cuisse droite qui comprend la moitié du genou ${ }^{14}$ », indépendant au moment de la découverte, avait permis à François Rever de repérer une particularité technique, sans comparaison à l'époque, qu'il décrivit et illustra graphiquement dès 1823 : des lacunes liées à d'importants défauts de coulée avaient été comblées par des plaques découpées de manière irrégulière, maintenues à l'aide de rivets à tête ronde, et dans certains cas par des contreplaques au revers ${ }^{15}$. L'objet de cette réparure était de masquer les défauts d'aspect consécutifs à la fabrication de la statue : porosités et lacunes formées en raison de dégagements gazeux lors de la coulée, trous laissés par les clous distanciateurs et les armatures utilisés pour maintenir le noyau dans le moule, soudure trop visible, etc. La technique est ici atypique car les plaquettes, de forme polygonale comme souvent à la période romaine, sont de très grande taille. Du fait de ces dimensions hors norme, les plaquettes de l'Apollon de Lillebonne ont 
nécessité pour leur fixation sur la statue la pose d'une multitude de rivets. La même technique a également été identifiée récemment sur le relief de grands dauphins dorés de Vienne (Isère) ${ }^{16}$ et sur les fragments d'une ou de plusieurs statues en bronze doré, mis au jour en 2008 à Horbourg-Wihr (Alsace) ${ }^{17}$. Si la répartition géographiquement limitée des occurrences se confirme à la lumière de nouvelles découvertes, une telle particularité pourrait être interprétée comme une signature gallo-romaine.

\section{Restauration}

Il est difficile de préciser la nature de l'intervention effectuée sur la statue durant son court premier séjour à Paris ${ }^{18}$. Nous savons en revanche qu'elle n'a pas été touchée à Londres, Woodburn ayant refusé les propositions qui lui ont été faites ${ }^{19}$. C'est à son arrivée au Louvre, à la fin de l'année 1853, qu'elle a subi une restauration importante pour son exposition au musée (introduction d'une armature interne fixée dans un socle polygonal, restitution en plâtre de la jambe droite sous le genou, comblements des lacunes au plâtre également). Des inquiétudes suscitées par l'instabilité de l'œuvre sur son socle ont conduit à une nouvelle intervention en 1942-1943. Il a fallu cependant attendre le démontage total de la statue, avant son traitement au Laboratoire d'archéologie des métaux à Jarville en 1974-1975, sous la direction d'Albert FranceLanord, pour comprendre les étapes antérieures et pallier le caractère sommaire des informations recueillies dans les $\operatorname{archives}^{20}$.

\section{La statue de culte d'un sanctuaire gallo-romain}

Apollon, divinité salutaire en Gaule, tenait une lyre dans la main gauche selon un schéma particulièrement prisé dans le Nord et le Centre-Est. L'effigie divine évoque par sa pondération et ses proportions générales l'influence des créations grecques du $\mathrm{IV}^{\mathrm{e}}$ siècle avant J.-C. sur le sol gaulois. L'équilibre approximatif de la figure, l'attache inorganique des membres à un torse étroit, la mollesse de la musculature et le caractère féminin de la coiffure comme des traits du visage témoignent toutefois d'une approche stylistique classicisante propre à la Gaule romaine, illustrée notamment par les Apollons du Vieil-Évreux et de Vaupoisson ${ }^{21}$, ainsi que par une statuette en bronze doré exhumée à Reichshoffen ${ }^{22}$. Le bronze a été produit au $\mathrm{II}^{\mathrm{e}}$ siècle de notre ère, possiblement sous le règne d'Hadrien ou au début de l'époque antonine ${ }^{23}$. Lors de sa présentation temporaire au musée départemental de Rouen en 2015, dans le cadre de l'exposition sur Juliobona ${ }^{24}$, il est apparu en parfaite cohérence avec l'environnement artistique que lui offraient les vestiges du décor sculpté du théâtre, les stèles funéraires des nécropoles de Lillebonne et quelques sculptures. Dans une lettre datée du 10 février 1825, Holley évoquait les objets mis au jour sur son terrain après la découverte de l'Apollon. Les deux statuettes votives - l'une de Dispater-Sucellus et l'autre d'un dieu Lare - et une lampe à bec double ornée, sur le réservoir, d'un Amour chevauchant un dauphin ont été dessinées par Langlois ${ }^{25}$. La présence dans le dépôt d'une monnaie de bronze à l'effigie de Julia Domna date l'enfouissement postérieurement aux années 211-218. Le terrain Holley se trouvait sans doute à l'emplacement d'un sanctuaire dont l'effigie cultuelle était la statue d'Apollon ${ }^{26}$. Les motifs de l'ensevelissement du bronze nous échappent. Toutefois, l'état relativement complet de l'œuvre indique qu'elle n'a pas été détruite rituellement dans un contexte d'abandon de sanctuaire, mais qu'elle a plutôt été endommagée lors d'événements politiques puis enfouie pour sa préservation ${ }^{27}$.

\section{L'identification d'un fragment isolé et la question de la composition du bronze}

La libéralité avec laquelle de petits fragments de la statue ont été distribués s'explique par le sentiment exprimé par François Rever, dès le 5 août 1823, dans une lettre au préfet : «Il y a beaucoup de petits fragments au raccordement desquels je tiens pour certain qu'il ne faut pas songer ${ }^{28}$. » Outre les sept petits morceaux de la statue parvenus en 1838 et 1840 au musée départemental des Antiquités de Rouen, cinq fragments ayant appartenu à François Rever ont été retrouvés en 1958 dans les réserves du musée d'Évreux et demandés pour le Louvre par Jean Charbonneaux, le conservateur en chef du département des Antiquités grecques et romaines du musée ${ }^{29}$. Ils ne sont pas localisés aujourd'hui. Un fragment a cependant été récemment identifié dans les réserves du musée. Il avait toute chance d'appartenir à la statue à cause d'une étiquette portant l'inscription «Bronze doré de la statue de l'Angleterre ", une mention manuscrite vraisemblablement rédigée durant les années de séjour de l'Apollon à Londres (fig. 3$)^{30}$.

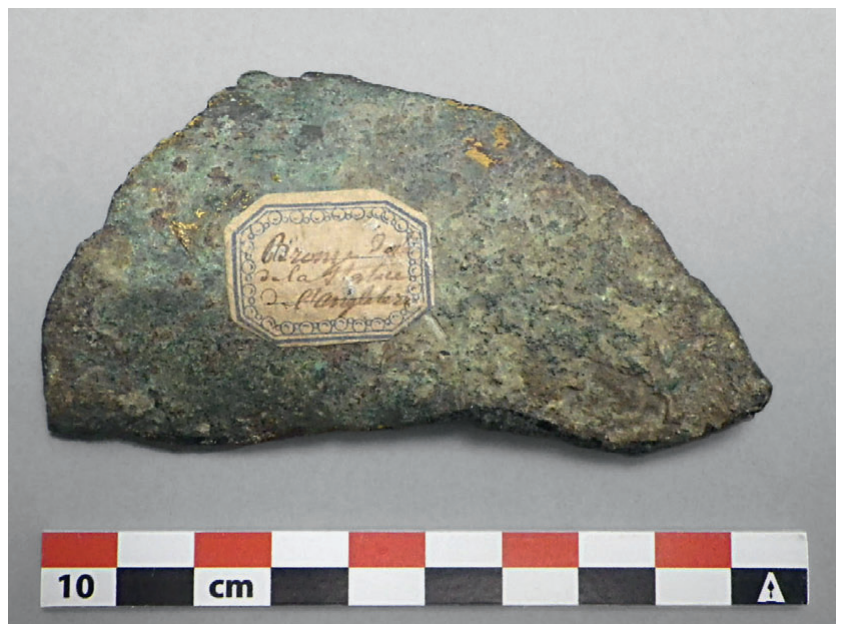

Fig. 3. Apollon de Lillebonne, fragment isolé portant la mention manuscrite : "Bronze doré de la statue de l'Angleterre ", musée du Louvre, département des Antiquités grecques, étrusques et romaines, Br 37.1. (C) C2RMF/A. Chauvet. 
Dès l'année de la découverte, François Rever sollicite de grands chimistes de l'époque et leur confie quelques fragments, dans le but de déterminer la composition du bronze, de décrire l'altération du métal et de discuter la technique de dorure. Il prend une part très active dans les discussions, n'oublions pas qu'il était le fondateur de l'école centrale de l'Eure, dans laquelle il enseignait la physique et l'histoire naturelle ${ }^{31}$. Les premières analyses sont effectuées en septembre 1823 par François Joseph Houtou Labillardière, professeur de chimie à l'université de Rouen, à partir de fragments que lui confie Rever. On devine dans la correspondance échangée qu'une véritable collaboration scientifique s'est engagée entre les deux hommes ${ }^{32}$; les résultats de ces analyses sont synthétisés par Houtou Labillardière dans une note annexée à la publication faite par Rever en $1824^{33}$. D'autres analyses ont été effectuées en 1824 par Louis-Nicolas Vauquelin, vraisemblablement au laboratoire des essais de l'hôtel de la Monnaie de Paris où il occupait la place de chef du Bureau de garantie ${ }^{34}$. Un premier fragment de 7 g confié par Rever fut tout d'abord analysé, puis un morceau de $15 \mathrm{~g}$ remis par Holley, lui-même divisé en 6 parties qui ont été analysées séparément ${ }^{35}$.

Les deux chimistes s'accordent sur le fait que le métal de la statue est fortement corrodé, et décrivent ce que l'on désigne aujourd'hui comme une corrosion intergranulaire : « L'intérieur [c'est-à-dire le métal vu en coupe] avait une teinte brune-rougeâtre, analogue à celle du cinabre en masse, et présentait des points brillants dans quelques-unes de ses parties ${ }^{36}$. » Leurs analyses ont été effectuées par dosage gravimétrique, en isolant un à un les différents constituants de l'alliage. Tous deux mettent en évidence un bronze contenant un peu de plomb : 5 à $6 \%$ d'étain et du plomb en quantité non mesurable selon Houtou Labillardière ${ }^{37}, 8 \%$ d'étain et $2 \%$ de plomb selon Vauquelin ${ }^{38}$.

Depuis 1824, la statue n'a pas fait l'objet d'autres études de sa technique de fabrication, hormis la dorure (voir ci-après). Toutefois, lors de la restauration effectuée en 1974-1975, deux fragments ont été envoyés par Albert France-Lanord au C2RMF, alors Laboratoire de Recherche des Musées de France (LRMF), où ils ont été analysés par Jacques Françaix. Les analyses avaient alors livré un résultat totalement différent de celles faites au XIX ${ }^{\mathrm{e}}$ siècle, puisqu'un alliage cuivreux quaternaire avait été mis en évidence ( $\mathrm{Zn}: 4,5 \%$; Sn : 3,4\% ; $\mathrm{Pb}: 3,0 \%)^{39}$. Nous avons déjà souligné ailleurs à quel point ce résultat était surprenant, faisant de l'Apollon de Lillebonne le seul grand bronze d'époque romaine allié au zinc ${ }^{40}$.

La redécouverte d'un fragment dans les réserves du musée du Louvre ${ }^{41}$ et les résultats contradictoires obtenus aux XIX ${ }^{\mathrm{e}}$ et $\mathrm{XX}^{\mathrm{e}}$ siècles rendaient indispensables la réalisation de nouvelles analyses. Nous avons pour cela procédé à des microprélèvements par forage $(1 \mathrm{~mm}$ de diamètre, 5 à $10 \mathrm{~mm}$ de profondeur), directement sur la statue pour disposer de compositions de référence, ainsi que sur le fragment retrouvé afin de pouvoir tester l'hypothèse de son appartenance à l'œuvre. Au total, neuf analyses ont été effectuées : jambe gauche, bras gauche, bras droit, tête, corps (coulées primaires), une plaquette polygonale et un de ses rivets (réparure) ; et pour le fragment, dans la partie correspondant à la coulée primaire, et dans celle d'une coulée secondaire (soudure ou réparure). Comme nos illustres prédécesseurs, nous avons également fait face à la corrosion intergranulaire du métal, qui affecte en particulier les prélèvements effectués sur le bras gauche, sur la tête, et sur le fragment (coulée primaire).

L'analyse des prélèvements a été effectuée par spectrométrie d'émission atomique par torche à plasma à couplage inductif (ICP-AES : Inductively Coupled Plasma Atomic - Emission Spectroscopy) au C2RMF. Nous avons choisi cette technique étant donné sa grande précision (environ $5 \%$ ) et sa très grande sensibilité (autour du ppm dans le métal, c'est-à-dire capable de détecter des teneurs de l'ordre de 0,0001\%). Pour chaque prélèvement, les copeaux ont été mis en solution dans l'eau régale (mélange concentré d'acides chlorhydrique et nitrique permettant de dissoudre tous les métaux). Les solutions obtenues ont été nébulisées dans le plasma d'argon produit par l'ICP-AES, il en a résulté une émission atomique dans l'ultraviolet et le visible qui a été mesurée : 29 éléments chimiques ont ainsi été simultanément dosés ${ }^{42}$. Préalablement à l'analyse, tous les prélèvements ont été contrôlés à la loupe binoculaire et, le cas échéant, les produits non métalliques ont été écartés. Ce n'est toutefois pas le cas des trois prélèvements affectés par la corrosion intergranulaire, pour lesquels corrosion et métal sont si intimement mélangés que le tri n'est pas envisageable.

Les résultats obtenus montrent une belle homogénéité de composition (voir tableaux la et 1b). Les différentes coulées primaires (tête, jambe, corps, bras) ont toutes été effectuées avec le même alliage, un bronze faiblement chargé en étain, $4,1 \%$ en moyenne ( $\min : 3,2 \%, \max : 4,5 \%$ ), et renfermant un peu de plomb $(3,2 \%$ en moyenne, min. $1,5 \%$; max. $5,8 \%)$. Le fragment de la «statue de l'Angleterre » s'intègre parfaitement dans ce schéma ( $\mathrm{Sn}: 4,1 \%$; $\mathrm{Pb}: 1,6 \%$ ). On remarquera que les trois analyses affectées par la corrosion intergranulaire sont également ceux pour lesquels le plomb est aux plus faibles teneurs. Il semble donc que la corrosion intergranulaire de l'Apollon de Lillebonne affecte principalement le plomb ; un contrôle par réalisation d'une coupe métallographique permettrait de le confirmer et donnerait peut-être la clé de l'histoire particulière de l'altération de la statue. Les coulées secondaires d'assemblage (soudures) qui unissaient les différentes coulées primaires n'ont pas été prélevées. Grâce au fragment découvert en réserve, une coulée secondaire (réparure ou soudure) est toutefois documentée, elle montre que le même alliage a été utilisé. Il en va de même pour la grande plaquette de réparure polygonale. L'unité de composition est confirmée par l'examen du spectre d'impuretés : il est particulièrement homogène, avec environ $0,1 \%$ d'antimoine et de fer, autour de $0,03 \%$ d'argent et d'arsenic, et $0,015 \%$ de nickel. Les rivets qui permettaient de maintenir les grandes plaquettes de réparure sont en revanche une notable exception, puisqu'ils sont faits en cuivre non allié. On observe de plus que ce cuivre renferme nettement moins d'impuretés. La même observation a été 
effectuée sur les grands dauphins de Vienne. L'intérêt de ce métal est évident pour la réalisation de rivets, le cuivre non allié est très plastique et s'écrase donc facilement au marteau pour constituer les têtes (arasées du côté extérieur). L'usage du cuivre non allié pose en revanche le problème de la couleur du métal : les rivets étaient beaucoup plus roses que la paroi de bronze. Cette technique de réparure n'est donc adaptée qu'aux bronzes destinés à être dorés.

Les nouvelles analyses confirment ainsi toutes les informations du XIX ${ }^{\mathrm{e}}$ siècle : un cuivre faiblement allié à l'étain et au plomb est à la base de la fabrication de l'Apollon de Lillebonne. Nos analyses permettent d'approcher beaucoup plus précisément sa composition : $4 \%$ d'étain et 3 à $6 \%$ de plomb. La corrosion intergranulaire n'est pas généralisée, mais doit affecter une grande partie de la statue. La raison de cette altération particulière devra être recherchée. Par ailleurs, le fragment récemment découvert en réserve ne laisse aucun doute, il provient bien de la statue. En revanche, on est en droit de s'interroger sur le fragment qui avait été isolé et analysé en 1975.

\section{L'étude de la dorure}

\section{Historique et contexte de l'étude}

Les recherches menées ces dernières années au C2RMF sur les grands bronzes antiques ont permis des avancées importantes tant sur les techniques de fonte et d'assemblage ${ }^{43}$ que sur celles de la dorure et de la polychromie métallique ${ }^{44}$. L'usage préférentiel de la dorure à la feuille sans préparation à la période romaine est aujourd'hui bien établi ${ }^{45}$, mais il existe encore de nombreuses incertitudes sur les techniques de mise en œuvre, les modules des feuilles utilisées ou leurs épaisseurs.

Comme nous l'avons mentionné ci-dessus, l'état de conservation exceptionnel de la dorure de l'Apollon avait frappé les esprits dès sa découverte. François Rever engagea lui-même des recherches : "La dissolution de deux petits fragments dans de l'acide nitrique, m'a fait obtenir, bien entières, les portions de feuilles d'or qui les recouvraient. [...] un troisième fragment d'une plus grande surface [...] m'a fourni neuf petites folioles de grandeurs différentes. [...] ces folioles rangées les unes à côté des autres eussent couvert une surface presque triple de celle du fragment. La dorure de ce fragment que le hasard m'avait offert avait sans doute été réparée ${ }^{46}$. » Par ailleurs, on découvrait les diverses propriétés de l'électricité, dont le phénomène de corrosion galvanique. Examinant la statue, Houtou Labillardière en conclut que la corrosion si particulière du bronze avait été provoquée par le couplage galvanique avec l'or de la dorure ${ }^{47}$. Il en naquit une controverse avec Vauquelin, qui, ayant examiné d'autres bronzes antiques dorés dont, en particulier, les chevaux de Saint-Marc lors de leur restauration à la Monnaie de Paris, réfutait le fait qu'un couplage galvanique puisse avoir causé

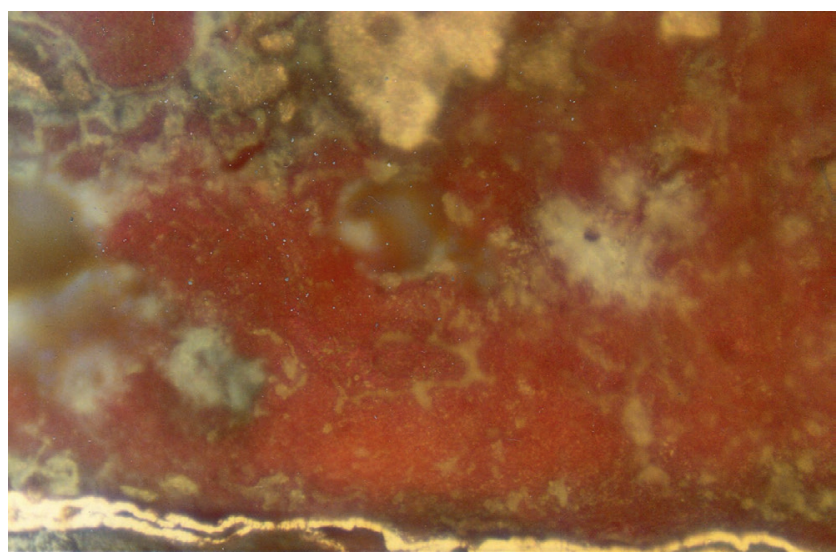

Fig. 4. Coupe métallographique de l'échantillon 129 (genou gauche) micrographie MB 95-7 (C) LAM/A. France Lanord.

une telle corrosion ${ }^{48}$. Les interventions successives de restauration allaient cependant se focaliser essentiellement sur les possibilités de nettoyage de la dorure plutôt que sur ses caractéristiques.

Plusieurs études partielles avaient toutefois abordé cette question, mais sans en tirer de conclusions définitives. Lors de l'étude et de la restauration menée en 1974-1975 par le Laboratoire d'archéologie des métaux ${ }^{49}$, Albert France-Lanord avait ainsi réalisé plusieurs coupes métallographiques, dont deux comportent de la dorure - échantillon 129 (genou gauche) avec les micrographies MB 95-9 et MB 95-7 (fig. 4) et échantillon 132 non localisé, avec la micrographie MB 94-1 ${ }^{50}$. La micrographie MB 95-9 montre une coupe métallographique prise à un grossissement de 400 fois avec la légende "métal presque entièrement corrodé, à g. la feuille de dorure couverte de carbonates ». La micrographie MB 95-7 montre la même coupe métallographique, mais pour une autre zone prise à un grossissement de 400 fois avec la légende «même échantillon, en bas la couche d'or formée de 2 feuilles ». La micrographie MB 94-1 montre une coupe métallographique prise à un grossissement de 100 avec la légende "métal entièrement corrodé. Quelques points de métal libre. À gauche, la feuille d'or couverte de carbonates". Il a donc bien été noté la présence dans un cas d'une couche d'or et, dans un autre, de deux feuilles distinctes, mais l'étude de la dorure n'a pas été approfondie. Il a surtout été question d'alliages et de phénomènes de corrosion. Ces coupes métallographiques ont été soigneusement conservées par le Laboratoire d'archéologie des métaux et ont pu être ré-exploitées à l'occasion de cette recherche complémentaire.

En 1990, dans le cadre d'une étude portant sur les dorures gallo-romaines et mérovingiennes ${ }^{51}$, Lucile Beck avait effectué des analyses à l'aide de l'accélérateur de particules AGLAE sur les fragments conservés à Rouen. Les analyses menées en PIXE (Particule Induced X-ray Emission) ont permis de donner une composition de la couche d'or, et celles en RBS (Rutherford Backscattering Spectrometry) une estimation de l'épaisseur de la feuille de l'ordre de 3 micromètres. Toutefois, les fragments analysés n'ayant pu être localisés sur 
Tableau 1a. Analyse ICP-AES de la composition élémentaire des alliages à base de cuivre de l'Apollon de Lillebonne (résultats en \% massique)

$\mathrm{CP}$ : coulée primaire ; $\mathrm{R}$ : réparure ; $\mathrm{CS}$ : coulée secondaire (assemblage ou réparure) ; nd : inférieur à la limite de détection ;

${ }^{\circ}$ : inférieur à la limite de quantification.

\begin{tabular}{|c|c|c|c|c|c|c|c|c|c|c|c|c|c|}
\hline $\mathrm{n}^{\circ}$ an. & $n^{\circ}$ inv & $\begin{array}{l}\text { Local. } \\
\text { prélèvement }\end{array}$ & Type & $\mathbf{C u}$ & Sn & $\mathbf{P b}$ & Ag & As & $\mathbf{F e}$ & $\mathbf{N i}$ & $\mathbf{S}$ & $\mathbf{S b}$ & $\mathbf{Z n}$ \\
\hline FZ1584a & Br 37 & $\begin{array}{l}\text { Jambe gauche, } \\
\text { sous le pied }\end{array}$ & $\mathrm{CP}$ & $\mathrm{X}$ & $\begin{array}{l}4,1 \\
\pm 0,4\end{array}$ & $\begin{array}{l}5,8 \\
\pm 0,6\end{array}$ & $\begin{array}{l}0,030 \\
\pm 0,003\end{array}$ & $\begin{array}{l}0,037 \\
\pm 0,004\end{array}$ & $\begin{array}{l}0,087 \\
\pm 0,009\end{array}$ & $\begin{array}{l}0,016 \\
\pm 0,002\end{array}$ & $\begin{array}{l}\text { nd } \\
<0,3\end{array}$ & $\begin{array}{l}0,10 \\
\pm 0,01\end{array}$ & $\begin{array}{l}0,0072 \\
\pm 0,0007\end{array}$ \\
\hline FZ1584b & Br 37 & $\begin{array}{l}\text { Bras gauche, } \\
\text { index }\end{array}$ & $\mathrm{CP}$ & $\mathrm{X}$ & $\begin{array}{l}4,2 \\
\pm 0,4\end{array}$ & $\begin{array}{l}3,3 \\
\pm 0,3\end{array}$ & $\begin{array}{l}0,027 \\
\pm 0,003\end{array}$ & $\begin{array}{l}0,039 \\
\pm 0,004\end{array}$ & $\begin{array}{l}0,080 \\
\pm 0,008\end{array}$ & $\begin{array}{l}0,016 \\
\pm 0,002\end{array}$ & $\begin{array}{l}\text { nd } \\
<0,3\end{array}$ & $\begin{array}{l}0,11 \\
\pm 0,01\end{array}$ & $\begin{array}{l}0,0067 \\
\pm 0,0007\end{array}$ \\
\hline FZ1584c & Br 37 & Tête & $\mathrm{CP}$ & $\mathrm{X}$ & $\begin{array}{l}4,5 \\
\pm 0,5\end{array}$ & $\begin{array}{l}5,0 \\
\pm 0,5\end{array}$ & $\begin{array}{l}0,029 \\
\pm 0,003\end{array}$ & $\begin{array}{l}0,033 \\
\pm 0,003\end{array}$ & $\begin{array}{l}0,11 \\
\pm 0,01\end{array}$ & $\begin{array}{l}0,015 \\
\pm 0,002\end{array}$ & $\begin{array}{l}0,51 \\
\pm 0,05\end{array}$ & $\begin{array}{l}0,10 \\
\pm 0,01\end{array}$ & $\begin{array}{l}0,0016 \\
\pm 0,0003\end{array}$ \\
\hline FZ1584d & Br 37 & Bras droit & $\mathrm{CP}$ & $\mathrm{X}$ & $\begin{array}{l}4,2 \\
\pm 0,4\end{array}$ & $\begin{array}{l}1,5 \\
\pm 0,1\end{array}$ & $\begin{array}{l}0,032 \\
\pm 0,003\end{array}$ & $\begin{array}{l}0,037 \\
\pm 0,004\end{array}$ & $\begin{array}{l}0,082 \\
\pm 0,008\end{array}$ & $\begin{array}{l}0,014 \\
\pm 0,001\end{array}$ & $\begin{array}{l}\text { nd } \\
<0,3\end{array}$ & $\begin{array}{l}0,11 \\
\pm 0,01\end{array}$ & $\begin{array}{l}0,0044 \\
\pm 0,0004\end{array}$ \\
\hline FZ1584e & Br 37 & Corps & $\mathrm{CP}$ & $X$ & $\begin{array}{l}3,2 \\
\pm 0,3\end{array}$ & $\begin{array}{l}2,2 \\
\pm 0,2\end{array}$ & $\begin{array}{l}0,026 \\
\pm 0,003\end{array}$ & $\begin{array}{l}0,036 \\
\pm 0,004\end{array}$ & $\begin{array}{l}0,083 \\
\pm 0,008\end{array}$ & $\begin{array}{l}0,011 \\
\pm 0,001\end{array}$ & $\begin{array}{l}\text { nd } \\
<0,3\end{array}$ & $\begin{array}{l}0,14 \\
\pm 0,01\end{array}$ & $\begin{array}{l}0,0094 \\
\pm 0,0009\end{array}$ \\
\hline FZ1584f & Br 37 & $\begin{array}{l}\text { Rivet plaquette } \\
\text { jambe droite }\end{array}$ & $\mathrm{R}$ & $\mathrm{X}$ & $\begin{array}{l}0,049 \\
\pm 0,011\end{array}$ & $\begin{array}{l}0,027 \\
\pm 0,003\end{array}$ & $\begin{array}{l}0,0095 \\
\pm 0,0010\end{array}$ & $\begin{array}{l}0,012 \\
\pm 0,001\end{array}$ & $\begin{array}{l}\text { nd } \\
<0,00012\end{array}$ & $\begin{array}{l}0,0072 \\
\pm 0,0007\end{array}$ & $\begin{array}{l}\text { nd } \\
<0,3\end{array}$ & $\begin{array}{l}0,083 \\
\pm 0,008\end{array}$ & $\begin{array}{l}\text { nd } \\
<0,00011\end{array}$ \\
\hline FZ1584g & Br 37 & $\begin{array}{l}\text { Plaquette } \\
\text { polygonale } \\
\text { (jambe droite) }\end{array}$ & $\mathrm{R}$ & $\mathrm{X}$ & $\begin{array}{l}3,2 \\
\pm 0,3\end{array}$ & $\begin{array}{l}2,2 \\
\pm 0,2\end{array}$ & $\begin{array}{l}0,026 \\
\pm 0,003\end{array}$ & $\begin{array}{l}0,037 \\
\pm 0,004\end{array}$ & $\begin{array}{l}0,060 \\
\pm 0,006\end{array}$ & $\begin{array}{l}0,014 \\
\pm 0,001\end{array}$ & $\begin{array}{l}\text { nd } \\
<0,3\end{array}$ & $\begin{array}{l}0,13 \\
\pm 0,01\end{array}$ & $\begin{array}{l}\text { nd } \\
<0,00015\end{array}$ \\
\hline FZ1584h & Br 37 ? & $\begin{array}{l}\text { Paroi } \\
\text { métallique } \\
\text { (fragment) }\end{array}$ & CP & $\mathrm{X}$ & $\begin{array}{l}4,1 \\
\pm 0,4\end{array}$ & $\begin{array}{l}1,6 \\
\pm 0,2\end{array}$ & $\begin{array}{l}0,041 \\
\pm 0,004\end{array}$ & $\begin{array}{l}0,036 \\
\pm 0,004\end{array}$ & $\begin{array}{l}0,072 \\
\pm 0,007\end{array}$ & $\begin{array}{l}0,010 \\
\pm 0,001\end{array}$ & $\begin{array}{l}\text { nd } \\
<0,3\end{array}$ & $\begin{array}{l}0,11 \\
\pm 0,01\end{array}$ & $\begin{array}{l}0,0015 \\
\pm 0,0002\end{array}$ \\
\hline FZ1584i & $\operatorname{Br} 37$ ? & $\begin{array}{l}\text { Soudure } \\
\text { ou réparure } \\
\text { (fragment) }\end{array}$ & CS & $x$ & $\begin{array}{l}4,8 \\
\pm 0,5\end{array}$ & $\begin{array}{l}1,5 \\
\pm 0,2\end{array}$ & $\begin{array}{l}0,053 \\
\pm 0,005\end{array}$ & $\begin{array}{l}0,036 \\
\pm 0,004\end{array}$ & $\begin{array}{l}0,065 \\
\pm 0,007\end{array}$ & $\begin{array}{l}0,0063 \\
\pm 0,0006\end{array}$ & $\begin{array}{l}0,41 \\
\pm 0,04\end{array}$ & $\begin{array}{l}0,13 \\
\pm 0,01\end{array}$ & $\begin{array}{l}\text { nd } \\
<0,00014\end{array}$ \\
\hline
\end{tabular}

l'Apollon et n'étant plus accessibles aujourd'hui, l'intégration de ces résultats dans une nouvelle étude s'avérait problématique.

En 2010, de nouvelles analyses ont été effectuées par Marc Aucouturier et Jacques Castaing, directement sur la statue, à l'aide d'un équipement portable de spectrométrie de fluorescence $\mathrm{X}$. Les mesures, prises au niveau du torse, ont livré des épaisseurs de l'ordre de 3 micromètres également. Il était, là encore, difficile de préciser à quoi correspondaient réellement ces mesures même si les premières observations générales, conduites dans la salle d'exposition de l'œuvre, avaient conforté l'hypothèse d'une ou de plusieurs redorures antiques, sans toutefois pouvoir en cerner le périmètre avec certitude.

C'est à l'occasion de l'exposition organisée à Rouen en $2015^{52}$ que la décision a été prise d'engager une étude spécifique portant sur la dorure de l'Apollon puisqu'aucune synthèse n'avait encore été établie et que les questionnements demeuraient nombreux. S'il était net que l'Apollon avait été effectivement doré à la feuille, la technique de mise en œuvre était difficile à appréhender : l'organisation du chevauchement des feuilles d'or semblait peu cohérente et on pouvait s'interroger sur la présence d'une ou de plusieurs redorures, antiques ou modernes (fig. 8). Cette étude a été l'occasion d'utiliser diverses techniques - examens des surfaces en microscopie numérique 3D (fig. 5-6) ; mesures d'épaisseur par des méthodes non destructives en spectrométrie de fluorescence $\mathrm{X}$; réinterprétation des anciennes coupes métallographiques avec polissage ionique préalable.

\section{Observations préliminaires des états de surface}

Sur le plan de l'aspect général, l'œuvre présente certaines caractéristiques d'altérations tout à fait spécifiques. On constate en effet des corrosions plus importantes dans la partie supérieure du corps. Elles concernent la tête, le dessus des épaules et de l'avant-bras gauche fléchi : dans ces zones, la dorure a presque entièrement disparu et les surfaces 
Tableau 1b. Analyse ICP-AES de la composition élémentaire des alliages à base de cuivre de l'Apollon de Lillebonne (résultats en ppm massique)

Les éléments systématiquement sous la limite de détection n’ont pas été reportés dans le tableau :

$\mathrm{Ba}, \mathrm{Cd}, \mathrm{Cr}, \mathrm{Mg}, \mathrm{Mo}, \mathrm{Ti}, \mathrm{V}(<1 \mathrm{ppm}), \mathrm{G}, \mathrm{U}, \mathrm{W}(<20$ ppm).

\begin{tabular}{|c|c|c|c|c|c|c|c|c|c|c|c|c|}
\hline $\mathrm{n}^{\circ}$ an. & $n^{\circ}$ inv & Local. prélèvement & Type & $\mathrm{Au}$ & $\mathbf{B i}$ & Co & $\mathrm{Hg}$ & In & Mn & $\mathbf{P}$ & Se & Te \\
\hline FZ1584a & Br 37 & $\begin{array}{l}\text { Jambe gauche, } \\
\text { sous le pied }\end{array}$ & $\mathrm{CP}$ & $\begin{array}{l}3,2 \\
\pm 0,3\end{array}$ & $\begin{array}{l}34 \\
\pm 8\end{array}$ & $\begin{array}{l}5,1 \\
\pm 0,5\end{array}$ & $\begin{array}{l}6,2 \\
\pm 0,8\end{array}$ & $\begin{array}{l}7,9^{\circ} \\
<8,5 \\
>2,5\end{array}$ & $\begin{array}{l}\text { nd } \\
<0,1\end{array}$ & $\begin{array}{l}\text { nd } \\
<18\end{array}$ & $\begin{array}{l}27 \\
\pm 5\end{array}$ & $\begin{array}{l}40 \\
\pm 5\end{array}$ \\
\hline FZ1584b & Br 37 & Bras gauche, index & $\mathrm{CP}$ & $\begin{array}{l}3,1 \\
\pm 0,3\end{array}$ & $\begin{array}{l}18^{\circ} \\
<28 \\
>8\end{array}$ & $\begin{array}{l}5,7 \\
\pm 0,6\end{array}$ & $\begin{array}{l}5,9 \\
\pm 1,1\end{array}$ & $\begin{array}{l}9,1 \\
\pm 1,5\end{array}$ & $\begin{array}{l}\text { nd } \\
<0,1\end{array}$ & $\begin{array}{l}\text { nd } \\
<19\end{array}$ & $\begin{array}{l}25 \\
\pm 2\end{array}$ & $\begin{array}{l}44 \\
\pm 5\end{array}$ \\
\hline FZ1584c & Br 37 & Tête & $\mathrm{CP}$ & $\begin{array}{l}2,5 \\
\pm 0,3\end{array}$ & $\begin{array}{l}24^{\circ} \\
<30 \\
>9\end{array}$ & $\begin{array}{l}3,6 \\
\pm 0,7\end{array}$ & $\begin{array}{l}6,1 \\
\pm 0,6\end{array}$ & $\begin{array}{l}9,8 \\
\pm 2,0\end{array}$ & $\begin{array}{l}\text { nd } \\
<0,1\end{array}$ & $\begin{array}{l}\text { nd } \\
<20\end{array}$ & $\begin{array}{l}20 \\
\pm 2\end{array}$ & $\begin{array}{r}47 \\
\pm 5\end{array}$ \\
\hline FZ1584d & Br 37 & Bras droit & $\mathrm{CP}$ & $\begin{array}{l}3,0 \\
\pm 0,4\end{array}$ & $\begin{array}{l}15^{\circ} \\
<27 \\
>8\end{array}$ & $\begin{array}{l}4,8 \\
\pm 0,5\end{array}$ & $\begin{array}{l}6,3 \\
\pm 0,9\end{array}$ & $\begin{array}{l}5,1^{\circ} \\
<8,6 \\
>2,6 \\
\end{array}$ & $\begin{array}{l}\text { nd } \\
<0,1\end{array}$ & $\begin{array}{l}\text { nd } \\
<18\end{array}$ & $\begin{array}{l}22 \\
\pm 4\end{array}$ & $\begin{array}{l}42 \\
\pm 7\end{array}$ \\
\hline FZ1584e & Br 37 & Corps & CP & $\begin{array}{l}2,2 \\
\pm 0,4\end{array}$ & $\begin{array}{l}23^{\circ} \\
<24 \\
>7\end{array}$ & $\begin{array}{l}6,3 \\
\pm 0,6\end{array}$ & $\begin{array}{l}5,7 \\
\pm 0,6\end{array}$ & $\begin{array}{l}12 \\
\pm 2\end{array}$ & $\begin{array}{l}1,4 \\
\pm 0,1\end{array}$ & $\begin{array}{l}\text { nd } \\
<16\end{array}$ & $\begin{array}{l}46 \\
\pm 5\end{array}$ & $\begin{array}{r}43 \\
\pm 4\end{array}$ \\
\hline FZ1584f & Br 37 & $\begin{array}{l}\text { Rivet plaquette } \\
\text { jambe droite }\end{array}$ & $\mathrm{R}$ & $\begin{array}{l}\text { nd } \\
<0,4\end{array}$ & $\begin{array}{l}14^{\circ} \\
<22 \\
>7\end{array}$ & $\begin{array}{l}\text { nd } \\
<0,2\end{array}$ & $\begin{array}{l}6,4 \\
\pm 1,1\end{array}$ & $\begin{array}{l}3,9^{\circ} \\
<7,1 \\
>2,1\end{array}$ & $\begin{array}{l}\text { nd } \\
<0,1\end{array}$ & $\begin{array}{l}22^{\circ} \\
<49 \\
>15\end{array}$ & $\begin{array}{r}48 \\
\pm 5\end{array}$ & $\begin{array}{r}44 \\
\pm 8\end{array}$ \\
\hline FZ1584g & $\operatorname{Br} 37$ & $\begin{array}{l}\text { Plaquette polygonale } \\
\text { (jambe droite) }\end{array}$ & $\mathrm{R}$ & $\begin{array}{l}2,2 \\
\pm 0,3\end{array}$ & $\begin{array}{l}11^{\circ} \\
<30 \\
>9\end{array}$ & $\begin{array}{l}5,4 \\
\pm 0,5\end{array}$ & $\begin{array}{l}5,9 \\
\pm 1,1\end{array}$ & $\begin{array}{l}11 \\
\pm 3\end{array}$ & $\begin{array}{l}\text { nd } \\
<0,1\end{array}$ & $\begin{array}{l}51^{\circ} \\
<67 \\
>20\end{array}$ & $\begin{array}{l}29 \\
\pm 3\end{array}$ & $\begin{array}{l}50 \\
\pm 5\end{array}$ \\
\hline FZ1584h & Br $37 ?$ & $\begin{array}{l}\text { Paroi métallique } \\
\text { (fragment) }\end{array}$ & $\mathrm{CP}$ & $\begin{array}{l}3,4 \\
\pm 0,5\end{array}$ & $\begin{array}{l}32 \\
\pm 4\end{array}$ & $\begin{array}{l}3,6 \\
\pm 0,4\end{array}$ & $\begin{array}{l}6,1 \\
\pm 0,6\end{array}$ & $\begin{array}{l}8,2^{\circ} \\
<8,7 \\
>2,6\end{array}$ & $\begin{array}{l}\text { nd } \\
<0,1\end{array}$ & $\begin{array}{l}27^{\circ} \\
<60 \\
>18\end{array}$ & $\begin{array}{l}25 \\
\pm 3\end{array}$ & $\begin{array}{r}44 \\
\pm 4\end{array}$ \\
\hline FZ1584i & $\operatorname{Br} 37 ?$ & $\begin{array}{l}\text { Soudure ou réparure } \\
\text { (fragment) }\end{array}$ & CS & $\begin{array}{l}4,3 \\
\pm 0,4\end{array}$ & $\begin{array}{l}30 \\
\pm 4\end{array}$ & $\begin{array}{l}24 \\
\pm 2\end{array}$ & $\begin{array}{l}5,3 \\
\pm 0,7\end{array}$ & $\begin{array}{l}10 \\
\pm 1\end{array}$ & $\begin{array}{l}\text { nd } \\
<0,1\end{array}$ & $\begin{array}{l}\text { nd } \\
<18\end{array}$ & $\begin{array}{l}23 \\
\pm 2\end{array}$ & $\begin{array}{l}43 \\
\pm 6\end{array}$ \\
\hline
\end{tabular}

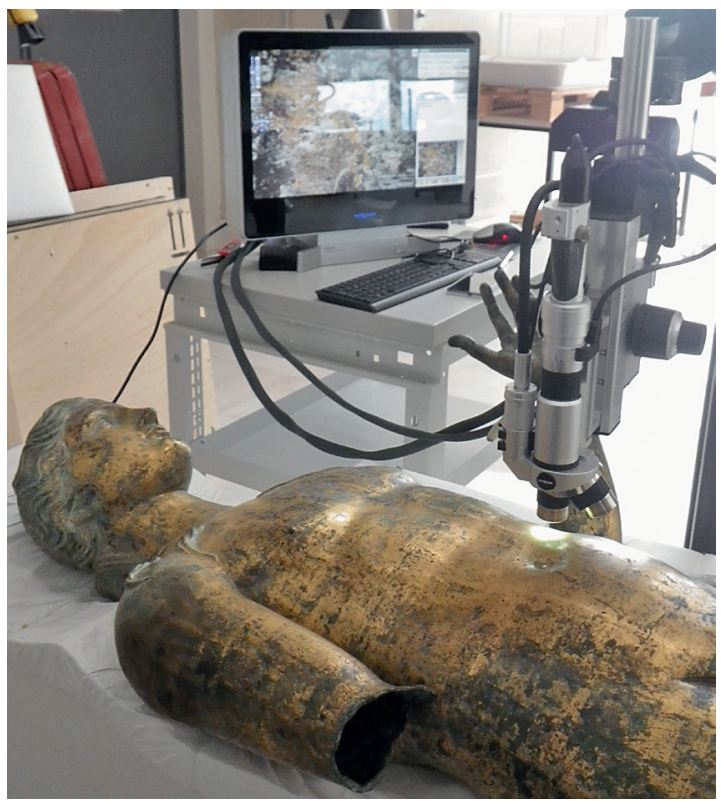

Fig. 5. La statue en cours d'examen en microscopie numérique 3D. (C) C2RMF/D. Robcis.

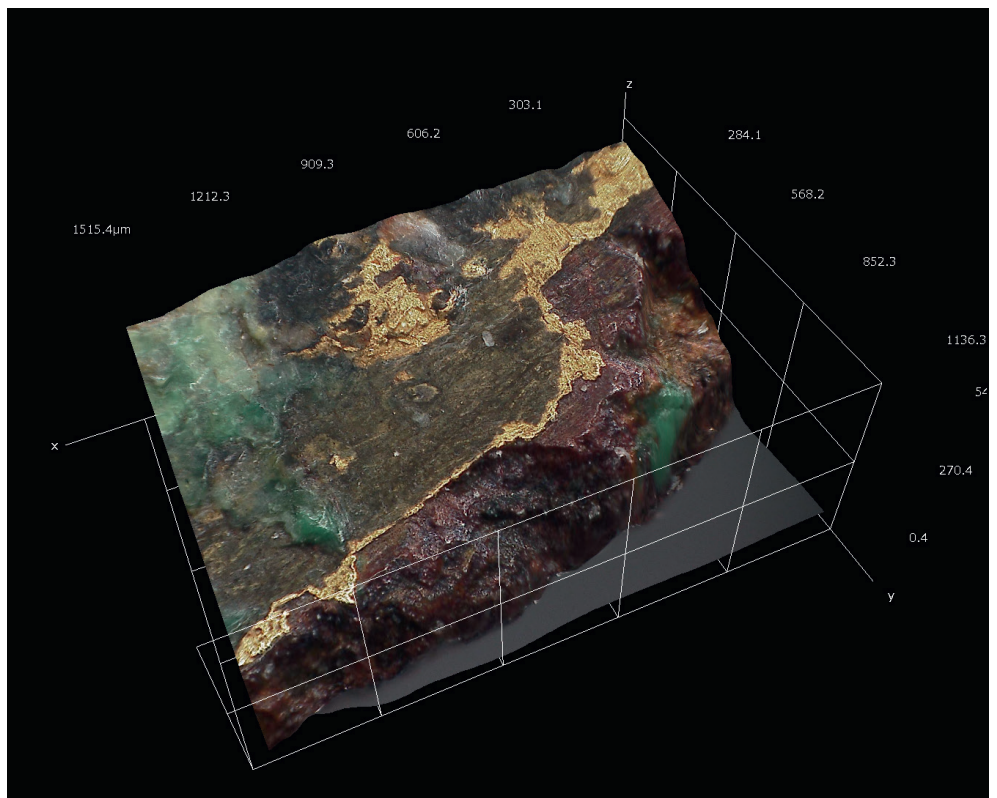

Fig. 6. Stratigraphie en imagerie numérique du fragment $\mathrm{Br}$ 37.1. En brun rouge, le bronze corrodé, recouvert par la dorure, elle-même surmontée d'une fine couche brune et d'amas de produits verts de corrosion du cuivre (reconstruction en 3D de surface). () C2RMF/D. Robcis. 


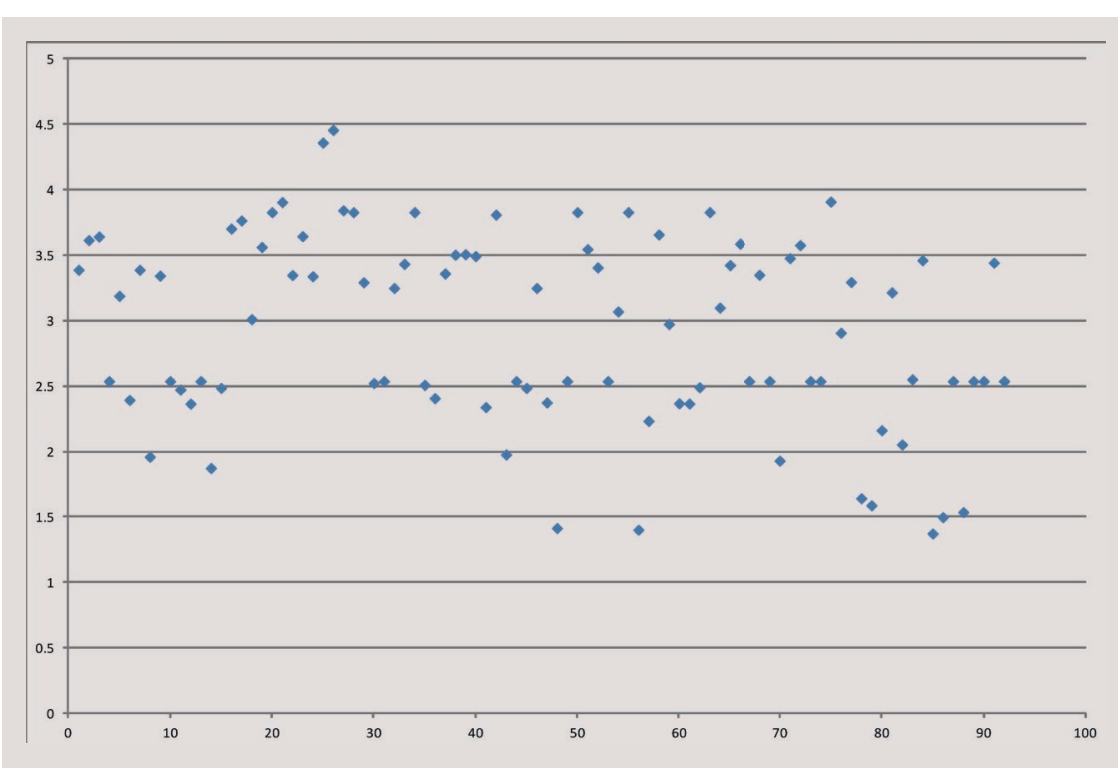

Fig. 7. Distribution des épaisseurs de dorure relevées sur la face antérieure de la statue en spectrométrie de fluorescence $\mathrm{X}$ (échelle micrométrique). corrodées présentent un aspect très érodé proche de ceux que l'on connaît pour les bronzes du XIX ${ }^{\mathrm{e}}$ siècle exposés à l'extérieur. Les zones généralement les mieux préservées sur de telles œuvres sont celles en dévers. On retrouve ces caractéristiques ici, sur le cou, et les parties inférieures des bras ; le dos semble beaucoup mieux préservé. Ce constat irait dans le sens d'une présentation antique en extérieur, sans doute à l'aplomb d'un mur. Ce point est particulièrement important, puisque les dorures à la feuille sont assez fragiles et résistent mal aux intempéries. Il fallait donc envisager le renouvellement régulier d'une protection de surface. Cela pouvait être un argument en faveur de plusieurs redorures successives, d'autant que le climat normand de Lillebonne se caractérise par des précipitations assez abondantes.

Les états de surface observables aujourd'hui ont subi les effets des nettoyages opérés sur la dorure lors des précédentes restaurations. Les techniques de nettoyage alors utilisées n'ont été que très partiellement documentées. L'état initial de l'œuvre lors de sa découverte n'est pas connu, ce qui rend particulièrement difficiles l'étude de la dorure et la compréhension des phénomènes d'altérations.

\section{Les analyses d'épaisseurs en spectrométrie de fluorescence $X$}

Afin d'estimer les épaisseurs et les éventuels chevauchements de feuilles - il fallait comprendre et vérifier la cohérence des réseaux -, une campagne d'analyses en spectrométrie de fluorescence X a été menée, avec plus de 100 points mesurés directement sur l'œuvre, sans prélèvements. Cette technique d'estimation des épaisseurs de revêtement en fonction du substrat est bien cernée par la littérature ${ }^{53}$ et des campagnes d'analyses précédentes conduites au C2RMF ont permis d'apprécier la corrélation entre les mesures effectuées à l'accélérateur en RBS et avec l'équipement de fluorescence X portable sur des standards d'or et sur des œuvres ${ }^{54}$. Il en était ressorti que les mesures par SFX sont corrélées à plus de $90 \%$ à celles effectuées en RBS, au moins dans la gamme d'épaisseurs allant de 0,2 à 10 micromètres. Dans le cas présent, nous sommes partis d'une matrice cuivre sur laquelle nous avons simulé une couche de plomb avant la couche d'or : en effet, dans le cas de l'Apollon, il s'agit d'un alliage ternaire contenant des taux de plomb assez élevés en surface (de l'ordre de $8 \%$ à $10 \%$ massique) qui risquaient de fausser les estimations.

Dans un premier temps, des mesures ont été effectuées sur la sculpture couchée et se sont donc limitées à la partie antérieure du corps. Ont pu être distinguées deux familles d'épaisseurs clairement différenciées, avec une concentration spécifique aux alentours de 2,5 micromètres qui correspond aux zones analysées dans le centre des feuilles et une autre, plus diffuse autour de 3,5 micromètres pour les zones de chevauchement (fig. 7). Il faut toutefois préciser que les mesures ont été faites sur des surfaces dégradées, et donc qu'il s'agit d'épaisseurs a minima. D'autre part, dans le cas de phénomènes de redorure, il est évident que l'on ne mesure pas l'épaisseur d'une feuille, mais sans doute l'épaisseur de deux feuilles dégradées dans leur partie centrale. De plus, dans les zones de chevauchement associées aux bords de feuilles, on a pu mesurer l'équivalent de cinq épaisseurs. Il a donc fallu interpréter ces résultats avec d'autres techniques d'analyse. Néanmoins, on peut d'ores et déjà retenir cette différence relativement constante de l'ordre de 1 micromètre $( \pm 0,2)$ entre les deux familles pour la partie antérieure.

\section{Format des feuilles d'or et techniques de pose}

Un relevé exhaustif, sur le torse et le ventre, des bandes en surface, assimilables à des zones de chevauchement, a permis de différencier deux formats de feuilles d'or, l'un de l'ordre 
de $11 \mathrm{~cm}$ au carré et l'autre de l'ordre de $9 \mathrm{~cm}$. Ces observations de modules différents se sont confirmées dans les autres parties du corps. On remarque en outre que la largeur des zones de chevauchement est différente selon ces modules : de l'ordre de $10 \mathrm{~mm}$ dans le cas des feuilles de $11 \mathrm{~cm}$, et de l'ordre de $5 \mathrm{~mm}$ dans l'autre cas. Il s'agit donc d'un système différent de mise en ouvre lors de la pose, confirmant l'hypothèse d'une redorure et précisant son périmètre.

Le système de pose des feuilles de grand format $(11 \mathrm{~cm})$ est très géométrique et régulier, orienté selon l'axe du corps. Le second système $(9 \mathrm{~cm})$, même s'il reste géométrique, est beaucoup moins régulier et est un peu désaxé par rapport au premier (fig. 9). Ce sont donc deux pratiques de pose des feuilles, bien différenciées par leur organisation spatiale et par la manière dont les chevauchements ont été opérés. À ce stade de l'étude, il n'était pas malheureusement pas possible de préciser quel système était antérieur à l'autre. Seule l'étude stratigraphique d'une zone où les deux couches de dorure étaient conservées pouvait permettre d'y répondre, mais cela impliquait un prélèvement.

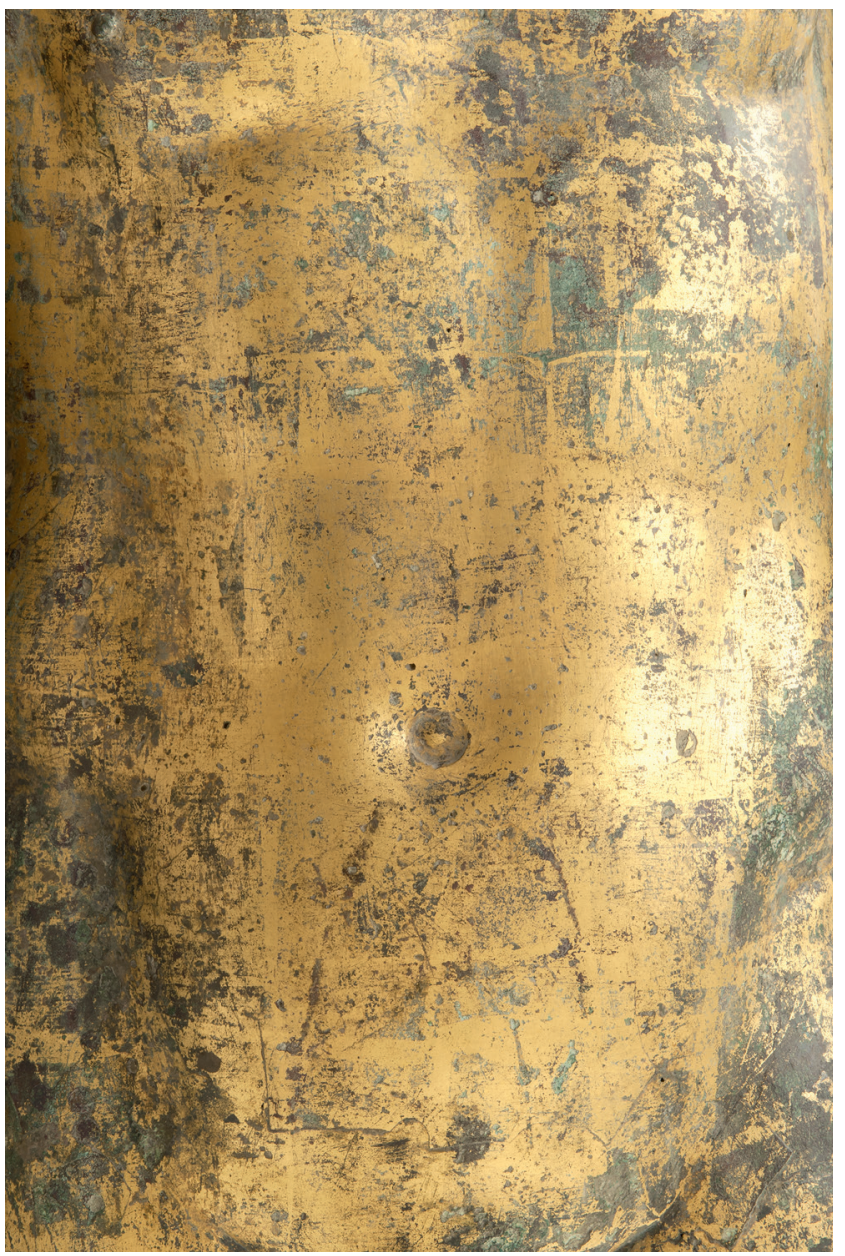

Fig. 8. Traces de chevauchements de feuilles d'or au niveau du torse. (c) C2RMF/A. Maigret.
À ce stade, il convient de noter par ailleurs que la dorure du dos, bien mieux conservée, n'offrait qu'un seul réseau de feuilles correspondant au grand module de $11 \mathrm{~cm}^{2}$, marqué par de larges zones de chevauchement. Les mesures effectuées en fluorescence $\mathrm{X}$ ont confirmé que nous avions bien une seule épaisseur, de l'ordre de 1,3 micromètre pour les cœurs de feuille, et d'environ 2,5 micromètres pour les zones de chevauchements. Ces observations ont donc permis d'envisager une chronologie relative de la dorure : une première phase a vu la mise en œuvre de feuilles de grand format, se chevauchant de manière large, tandis qu'une deuxième phase, limitée à la partie antérieure de l'œuvre, a vu la pose de feuilles de plus petit format, moins épaisses et avec un chevauchement beaucoup moins important. Il y aurait donc eu une campagne de redorure in situ, limitée à la face avant, ce qui suppose que la partie postérieure n'était pas ou était peu accessible. Ces déductions sont venues renforcer l'hypothèse d'une présentation de la statue contre un élément architectural, déjà formulée lors de l'observation de la corrosion.

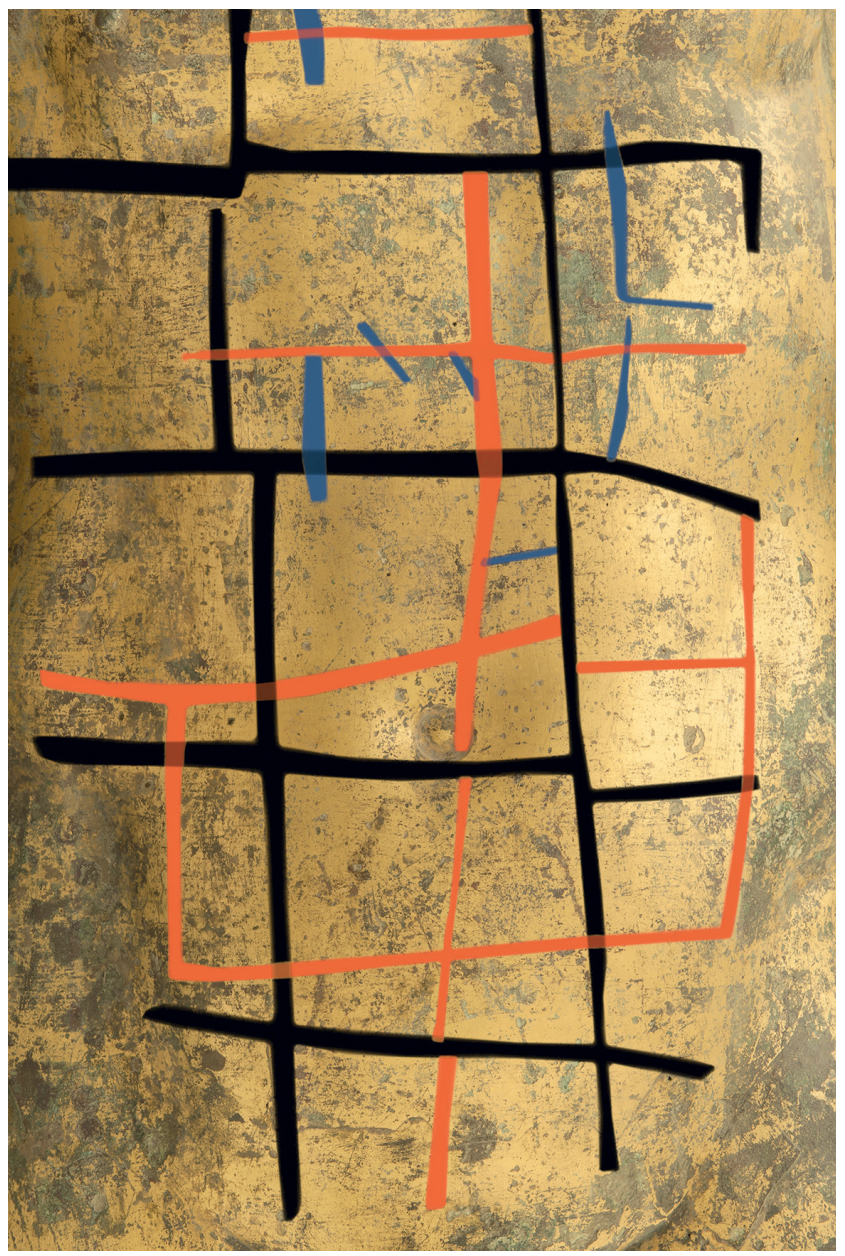

Fig. 9. Relevé des zones de chevauchements au niveau du torse ; en noir, le premier réseau de dorure et en rouge, le second, en bleu les éléments non résolus. (C) C2RMF/A. Maigret. 


\section{Nouvel examen des coupes métallographiques de 1975}

À l'issue de ces constatations, deux des coupes métallographiques réalisées par Albert France-Lanord (échantillons 129 et 132) ont fait l'objet d'une nouvelle étude avec reprise du polissage par abrasion et utilisation d'une technique de préparation spécifique par polissage ionique, suivie d'observations en microscopie électronique à balayage classique et à effet de champ. Cette technique de préparation permet d'éviter les risques d'écrasement de la feuille d'or lors du polissage, ce qui peut conduire à une surestimation de l'épaisseur, voire même du nombre de feuilles, si le polissage écrase les feuilles au point de les réunir. C'est d'ailleurs ce qui a pu être observé dans ce cas.

À l'examen de la coupe de l'échantillon 129 correspondant au genou (fig. 4), donc sur la face antérieure de la statue, reprise en polissage classique (fig. 10), on observe la présence de quatre feuilles d'or au lieu des deux notées par Albert France-Lanord. Ces quatre feuilles n'appartiennent en fait qu'à deux campagnes de dorure : les deux premières feuilles correspondent à une zone de chevauchement de la dorure initiale, alors que celles en surface correspondent à la phase de redorure. Les mesures effectuées sur les deux premières feuilles présentent une épaisseur variant entre $1,8 \mu \mathrm{m}$ et 2,5 $\mu \mathrm{m}$. Les deux feuilles situées au-dessus oscillent entre $1 \mu \mathrm{m}$ et $1,5 \mu \mathrm{m}$. Toutefois, comme il s'agit d'un polissage classique, les épaisseurs sont légèrement surestimées.

Le même échantillon a ensuite fait l'objet d'une préparation spécifique en polissage ionique, examinée à un grossissement de 11000 fois, dans le but de mieux cerner les épaisseurs. Dans la zone observée, différente de celle dont il vient d'être question, on distingue trois feuilles d'or, dont les

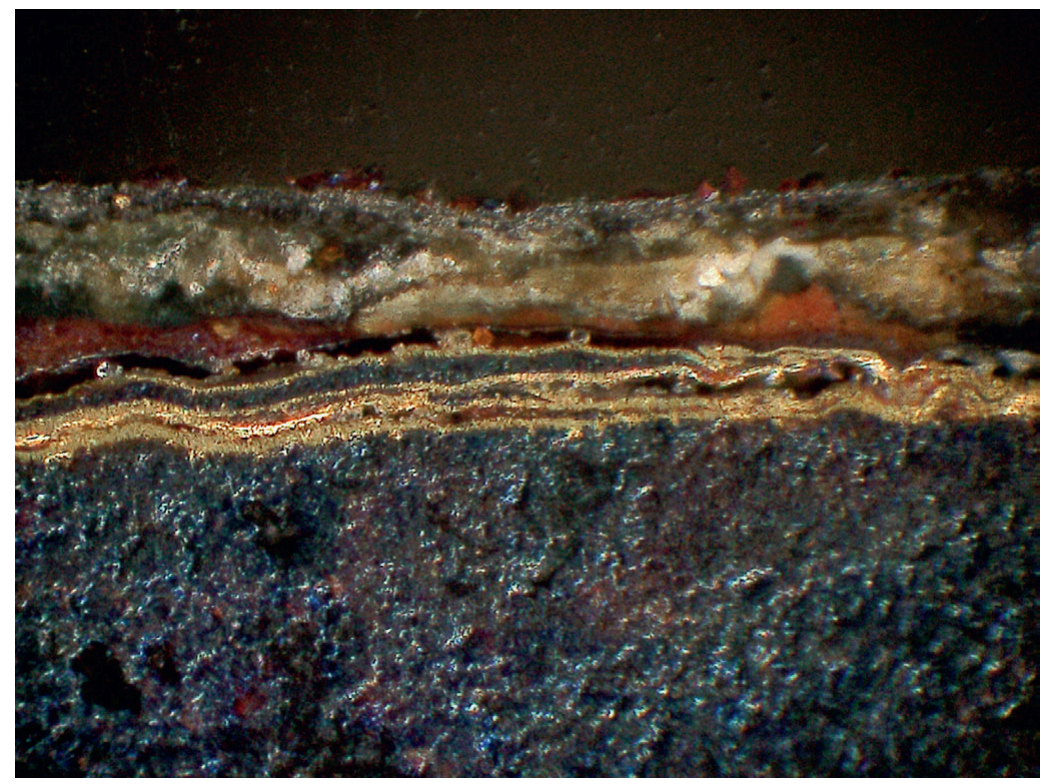

Fig. 10. Coupe métallographique MB 95-7 reprise en polissage classique, observation en microscopie numérique 3D à un grossissement de 400 fois. (C) C2RMF/D. Robcis.

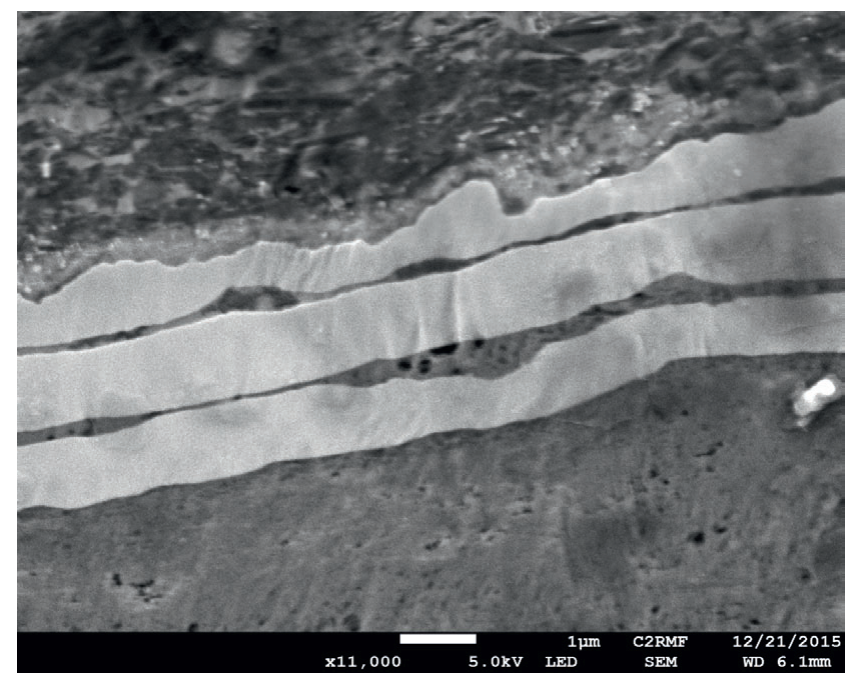

Fig. 11. Coupe métallographique MB 95-7 reprise en polissage ionique, observation en microscopie à effet de champ à un grossissement de 11000 fois. (c) C2RMF/N. Pingaud.

deux premières sont à peu près similaires et mesurent entre 0,8 et 1,2 micromètres (fig. 11). La dorure de surface est moins épaisse et beaucoup plus irrégulière, oscillant entre 0,5 et 1 micromètre. Nous sommes ici en présence d'une zone de chevauchement de la dorure initiale recouverte par la feuille de redorure.

Ces résultats viennent ainsi conforter les observations faites grâce aux relevés graphiques et aux mesures en spectrométrie de fluorescence X portable, mettant en évidence une première dorure relativement épaisse correspondant aux grands modules carrés de 11 centimètres, et une seconde, moins épaisse, correspondant aux petits module carrés de 9 centimètres, liée à la phase de redorure.

\section{Conclusion}

Dans la majorité des cas, les bronzes antiques érigés sur les places publiques et dans les sanctuaires n'étaient pas dorés. Il fallait tenter de préserver leur éclat et leur brillance en protégeant leur épiderme avec des substances huileuses et bitumineuses qui, si elles n'étaient pas périodiquement remplacées, modifiaient progressivement la teinte générale de l'alliage cuivreux ${ }^{55}$. Dorer un bronze était apparemment une manière antique de tenter de lui conserver l'apparence qui était la sienne au sortir de l'atelier ${ }^{56}$. Il est possible que la dorure ait elle-même reçu, dans le cas de l'Apollon, une protection de surface, cire ou vernis, car une fine couche brune a été observée sur l'épiderme en de nombreux endroits (fig. 12). Les analyses effectuées sur des prélèvements n'ont toutefois pas permis d'en préciser la nature ${ }^{57}$.

L'étude récente de l'Apollon démontre que cette statue de culte était très vraisemblablement exposée en plein air, contre un élément architectural, un mur peut-être ou une niche, qui protégeait son dos. Sa présentation n'a duré que durant quelques générations du $\mathrm{II}^{\mathrm{e}}$ siècle de notre ère, un 
Fig. 12. Fragment Br 37.1 : couche brune observée sur la surface de la dorure en microscopie numérique à un grossissement de 350 fois. (C) C2RMF/D. Robcis.

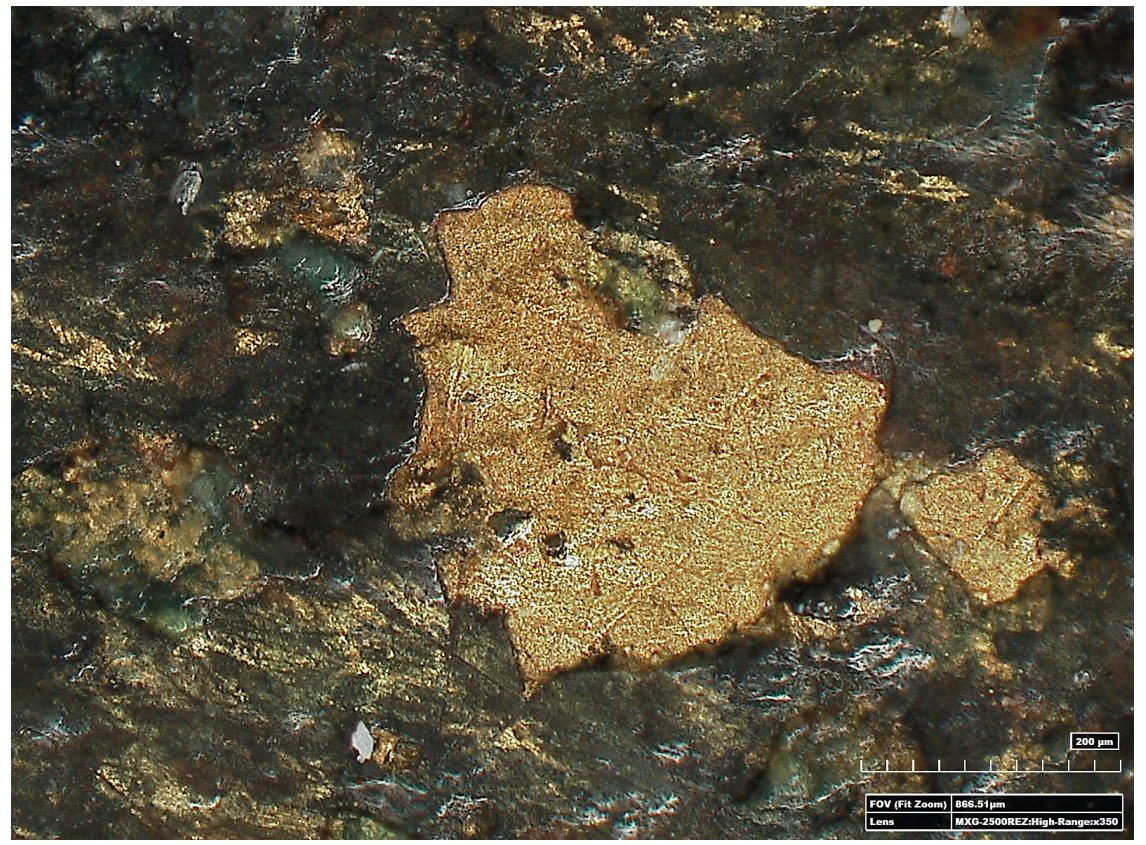

temps relativement court pendant lequel il a pourtant été redoré. Ce constat confirme l'importance accordée à l'entretien des œuvres jugées majeures ${ }^{58}$. Il faudrait vérifier sur d'autres bronzes dorés ${ }^{59}$ si cette pratique était exceptionnelle ou relativement courante. L'évolution des techniques d'examens et d'analyses, associée à celle des problématiques, a enrichi considérablement la connaissance technologique et historique de l'Apollon de Lillebonne. Cette étude confirme aussi que la compréhension d'un grand bronze antique repose nécessairement sur une approche collégiale.

\section{Notes}

1. Paris, musée du Louvre, département des Antiquités grecques, étrusques et romaines, inv. Br 37 (NIII 65), H. 1,94 m. Manquent l'avant-bras droit, l'auriculaire gauche, la jambe droite sous le genou, la face interne du genou gauche, le talon gauche et le sexe. De grandes lacunes affectent également l'omoplate droite et le bas du dos à gauche (Duval 1978, fig. 5-9a) Sur la période allant de la découverte de l'Apollon à son entrée au Louvre, voir Duval, 1978 , p. 265-267, 273-274 ; Tailliez, 1982 ; Dorion-Peyronnet, 2015. En sollicitant le prêt de l'Apollon à Rouen, Caroline Dorion-Peyronnet, commissaire en 2015 de l'exposition Lillebonne-Juliobona, nous a offert la possibilité de reprendre l'étude de la statue, laquelle a pu séjourner deux mois au C2RMF. Nous l'en remercions très sincèrement.

2. Il était le frère du maire de Lillebonne (lettres du comte de Clarac à M. de Cailleux et de Guillaume Léonard Holley au roi, Archives des musées nationaux, Pierrefitte, respectivement A6 1853, 30 septembre 1823 et A6 1824, 25 juillet 1833).

3. Lettre de Guillaume Chuinery à Alphonse de Cailleux, Archives des musées nationaux, Pierrefitte, A6 1853, 16 août 1823.

4. Dorion-Peyronnet, 2015, p. 20-24 fig. 11-12. Sur les fragments soumis en 1823 et 1824 à des analyses, voir notamment Rever, 1824, p. 20-24, 41-45 [Houtou La
Billardière pour ces cinq dernières pages] et Vauquelin, 1824.

5. Rever, 1824 , p. 5-29.

6. Le 22 août, à la demande de la commission des Antiquités de la SeineInférieure lors de sa séance du 13 août (Duval, 1978, p. 265)

7. La lettre, datée du 26 septembre 1823, est adressée au comte de Forbin, directeur général des Musées royaux,

Archives des musées nationaux, Pierrefitte, A6 1824.

8. Archives nationales, Pierrefitte, rapports au comte de Pradel pour l'année 1823, cote 0/3/1285. Que Mathilde

Vauquelin, qui nous a signalé ce document, trouve ici l'expression de notre

reconnaissance. Dans une lettre de synthèse des événements marquants depuis la découverte de la statue, établie par le comte de Forbin le 10 septembre 1833, il fut précisé que M. de Cailleux devait « tâcher d'entrer en négociation pour l'acquisition de la statue » et que de retour à Paris, il avait fait part au ministre de la Maison du roi « des prétentions exagérées du propriétaire », Archives des musées nationaux, Pierrefitte, A6 1824, 10 septembre 1833.

9. Archives des musées nationaux, Pierrefitte, A6 1853, 5 janvier 1824. Voir également Dorion-Peyronnet, 2015, p. 27.

10. Archives des musées nationaux, Pierrefitte, A6 1824, 31 octobre 1824. Dans une lettre du 11 novembre 1824 adressée à M. Holley, le vicomte de la Rochefoucault confirma qu'il renonçait à l'expertise, ayant suffisamment d'éléments pour juger du caractère inadéquat des offres (Archives nationales, Pierrefitte, fonds de la Maison du roi (monarchie de juillet, cote $0 / 3 / 1411$ ) documents également signalés par Mathilde Vauquelin.

11. Dorion-Peyronnet, 2015, p. 27.

12. Duval, 1978, p. 273, British Museum Library, Original Papers 1826-1828, lettre de Samuel Woodburn, datée du 8 mars 1828 ; Dorion-Peyronnet, 2015, p. 28 n. 79.

13. Archives des musées nationaux, Pierrefitte, A6 1853, 29 juillet 1853.

14. Rever, 1824 , p. 7

15. Rever, 1824, p. 12-14, pl. II-III, après la p. 45, avec légendes développées, sans pagination ; Duval, 1978, p. 269-272, fig. 3-4 ; Dorion-Peyronnet, 2015, p. 21 fig. 9 (relevé original sur calque des plaques rivetées)

Le « tronçon " comprend bien une partie du genou, comme indiqué sur le dessin de Langlois (fig. 2) et dans la lettre du comte de Clarac (ci-dessus n. 7).

16. http://c2rmf.fr/nos-activites/ programmes-termines/les-grands-dauphinsde-vienne (consultée le 19 mai 2017)

17. Roth-Zehner, Le Martret, Bailliot, 2015, p. 118-120, fig. 2. Fragments de statue (s) monumentale (s) liés à un habitat dont l'apogée se situe entre la deuxième moitié du II $^{\mathrm{e}}$ siècle et le milieu du III $^{\mathrm{e}}$ siècle, date de destruction de la zone.

18. Tailliez, 1982 , p. 82 et n. 20 : d'après 
la lettre de Bosio (ci-dessus n. 13), un praticien du nom de Liri aurait travaillé sur l'œuvre durant 28 jours. L'œuvre a séjourné dans l'atelier du sculpteur Rutxhiel.

Sur l'inquiétude de Rever quant à des restitutions abusives et sur la nature des interventions, voir Dorion-Peyronnet, 2015 . p. 27-28: on apprend par une lettre du 11 mai 1826, adressée à F. Rever par un certain Detonnaud, que la jambe manquante avait été remplacée par une jambe de cire.

19. Dans la lettre citée ci-dessus n. 12 "I had thought of having it restored before having it brought to England, but having seen wax models of the proposed restoration which did not class with the stile (sic) of the art of the bronze I have now brought it to England as discovered." Un dessin réalisé à Londres par J. G. Waller en 1853 (R. Smith, 1854 ; Duval, 1978, p. 274 ; Tailliez, 1982, p. 87, fig. 6) restitue la jambe et l'avant-bras droits sans ombres à la différence du sexe, traité comme le reste de la statue. Se pourrait-il qu'il ait été reconstitué à Paris avant le départ pour Londres? Il s'agit d'une adjonction métallique, maintenue en place par un écrou en fer (Duval, 1978, p. 276).

20. La seule information sur l'intervention de 1853 se résume à la mention suivante d'Adrien de Longpérier : «Je vous prie de hâter le plus possible l'achèvement de la statue de bronze de Lillebonne et le placement de cette figure dans la salle des bronzes ", Archives des musées nationaux, Pierrefitte, A6 1853, 15 novembre 1853. À l'exception de l'armature métallique interne, cette restauration demandée au responsable des marbriers a fait appel à du bois, des chiffons, du papier et du plâtre. Sur les différentes interventions, de 1853 à 1975, voir Duval, 1978, p. 274-283 : la figure 2 présente la statue après démontage en 1974 des parties restaurées. Plusieurs photographies, réalisées au Laboratoire d'archéologie des métaux, documentent l'existence d'une coulée de bronze effectuée au XIX ${ }^{\mathrm{e}}$ siècle (durant le premier séjour de la statue à Paris, avant son départ pour Londres? En 1853 ?), pour compléter le genou gauche lacunaire, une coulée qui englobait des fragments antiques. Ces photographies sont conservées dans le dossier de l'œuvre au Louvre.

21. Lenormant, 1875 ; Espérandieu, $1911, n^{\text {os }} 3063,3216$, p. 170-171, 263 ; Boucher, 1976, p. 129-131, 374, pl. 16. Sur le rapprochement des deux bronzes avec l'Apollon de Lillebonne, voir récemment Szewczyk 2013a, p. 20 ; Szewczyk, 2013b, p. 192, 196.

22. Schnitzler, $1995, \mathrm{n}^{\circ} 95$, p. 87.

23. Zanker, $1974, \mathrm{n}^{\circ}$ 6, p. 103-104.

24. Exp. Rouen 2015.

25. Rever, 1821, p. 128-141, pl. III bis. Pour comprendre pourquoi des statuettes trouvées en 1824 apparaissent dans une publication de 1821, voir en particulier p. 130: «J'avais envoyé depuis quelque temps à l'impression la dernière partie de mon Mémoire, lorsque j'appris que M. Holley venait de faire encore une découverte [...]. Les deux figures $[\ldots]$ proviennent du rejet même de la fosse d'où, l'année dernière, on exhuma la grande. " Espérandieu, Rolland, 1959, n ${ }^{\text {os }}$ 9, 65, 183 , p. 24, 44, 81, pl. II, XXVI, LV ; Rogeret, 1997 p. 384 fig. 377 ; Kaufmann-Heinimann, 1998, p. 252 : les deux statuettes ne sont pas localisées. La lampe est conservée au Römermuseum d'Augst.

Dorion-Peyronnet, 2015, p. 26, 29 : une lettre de Langlois adressée à François Rever le 14 juillet 1825 évoque aussi par des dessins et des commentaires une tête de marbre mise au jour le mois précédent « à l'endroit où fut découvert (sic) la grande statue en bronze doré en 1823 ». Ce portrait pourrait être celui de l'empereur Caracalla.

26. Kaufmann-Heinimann, 1998, p. 200. 27. Sur des témoignages de destructions rituelles de statues de bronze, voir Azéma t al., 2012, Azéma et al. (sous presse) pour les sanctuaires du Vieil-Évreux et de Genainville, et Kaurin et al. dans ce volume pour le guerrier de Saint-Maur, démonté avant d'être enfoui. La dégradation de l'Apollon est peut-être à mettre en relation avec les événements politiques des années 193-197, qui aboutirent à la victoire définitive de Septime-Sévère sur les autres candidats qui briguaient le pouvoir impérial (Rogeret, 1997, p. 328).

28. Dorion-Peyronnet, 2015, p. 20-23.

29. Lettre du conservateur du musée d'Évreux, J. M. Moulin, dans le dossier de l'Apollon. Il y est écrit que les cinq fragments étaient accompagnés de la mention «Morceaux de la statue d'Apollon en bronze doré trouvée à Lillebonne. Coll. de Fr. Rever ".

30. Ce fragment a reçu le numéro d'inventaire $\mathrm{Br}$ 37.1. L. $\max : 9,4 \mathrm{~cm}$, H. $\max .5,1 \mathrm{~cm}$.

31. Rever, 1802

32. Houtou Labillardière à Rever, 15 septembre 1823 : «Je me rappelai l'expérience que vous avez faite en calcinant la matière avec du charbon et dans laquelle vous vîtes paraître des vapeurs blanches qui dénotaient la présence d'arsenic ou de zinc. J'ai répété les essais dans le but de retirer la substance volatile par la calcination, et je n'ai pu rien volatiliser », Archives départementales de l'Eure, 47J155_fํ25492550 .

33. Analyse de quelques fragmens du métal de la Statue de Lillebonne, par M. Houtou la Billardière; Professeur de Chimie à Rouen, dans Rever, 1824, p. 41-45.

34. Bret, 2000.

35 . Vauquelin, 1824.

36. Houtou Labillardière dans Rever, 1824 , p. 41

37. Houtou Labillardière dans Rever, 1824, p. 42

38. Vauquelin, 1824, p. 398-399. Une note du Comte de Clarac rapporte l'épisode des analyses effectuées en 1823-1824, reprenant d'ailleurs de façon erronée les résultats obtenus par Houtou Labillardière et Vauquelin, mais ajoute surtout que des analyses ont été effectuées par J.-P.-J.

D'Arcet. Ni la date ni la référence de cette troisième série d'analyses ne sont données, seuls les résultats sont reportés : «M. D'Arcet a obtenu 82.513 de cuivre, 9.196 d'étain, 6.896 de plomb et 1.270 de zinc, et en outre 0.082 d'or et 0.042 d'argent, qui n'étaient qu'accidentellement alliés au bronze. ", Clarac, 1841, n. 2, p. 75-76. Les références à
Clarac et D’Arcet nous ont été communiquées par Brigitte Bourgeois. Nous l'en remercions vivement.

Sur la question des premières analyses chimiques d'œuvres antiques et les glorieux débuts de l'archéométrie française, voir Bourgeois, 2013

39. Françaix, voir au C2RMF le dossier FZ1584, rapport n ${ }^{\circ}$ Z527 du 25 août 1975, analyse par spectrométrie d'émission de quatre bronzes gallo-romains.

40. Mille et al., 2012, fig. 34.

41. Ci-dessus n. 30.

42. Pour plus de détails sur le protocole analytique par ICP-AES utilisé au C2RMF, consulter Bourgarit, Mille, 2003.

43. Voir par exemple Mille et al., 2012, sur les statues d'un enfant royal et d'Éros du Cap d'Agde.

44. Voir par exemple Aucouturier et al., 2004, pour la statue égyptienne de

Karomama.

45. Oddy, 2012.

46. Rever, 1824, p. 20-22.

47. Houtou Labillardière, 1824.

48. Vauquelin, 1824.

49. France-Lanord, 1975

50. Dans le dossier de l'œuvre au département des Antiquités grecques, étrusques et romaines, musée du Louvre.

51. Beck et al., 1991, p. 103-107.

52. Exp. Rouen, 2015

53. Cesareo, 2009.

54. Aucouturier, 2016.

55. Sur l'apparence, l'entretien et l'assombrissement progressif des bronzes, voir en dernier lieu Descamps-Lequime, 2015, p. 152-153, 159-160.

56. Descamps-Lequime, 2006, p. 84.

57. La Niké retrouvée dans le Rhône à Arles présentait dans certaines zones un état de surface comparable qui n'a pu être préservé en totalité. Voir Aucouturier et al., 2016, p. 187.

58. Sur la question générale de l'entretien de la sculpture dans l'Antiquité, voir Bourgeois (dir.), 2014.

59. Mentionnons, parmi les grands bronzes dorés représentant des divinités, la tête d'une statue de culte d'Apollon (Sofia, $\mathrm{II}^{\mathrm{e}}$ siècle, Institut archéologique national et musée, inv. 8483) ou une tête de SulisMinerve, découverte à Bath en 1727, qui a bien été redorée (Oddy et al. 1990, n 31, p. 112, 118-120, fig. 10a-10b).

\section{Bibliographie}

Aucouturier M., Delange E., Meyohas M.-E., 2004, « Karomama, Divine Adoratrice d'Amon : son histoire, sa restauration, l'étude en laboratoire ", Techné, 19, p. 7-16.

Aucouturier M., Robcis D., Langlois J., Vandenberghe Y., 2016, « La Victoire d'Arles : une dorure à la feuille exemplaire et complexe", dans S. Bouffier (dir.), La sculpture romaine en Occident : nouveaux regards, Errances, Arles, p. 181-190.

Azéma A., Mille B., Pilon F., Birolleau J.-C., Guyard L., 2012, «Étude archéométallurgique du dépôt de grands bronzes du sanctuaire gallo- 
romain du Vieil-Évreux (Eure)", Archéosciences, 36, p. 153-172.

Azéma A., Mille B., Vermeersch D., à paraître, "So many fragments of large bronze statues in Gallo-Roman sanctuaries in north-western France: the case of Genainville (Val d'Oise)", Actes de la conférence Wissenschaftliches Kolloquium Römische Großbronzen am UNESCO Welterbe Limes, 4-5/02/2015, Aalen (Germany), Willer F. (dir.).

Beck L., Beck F., Eluère C., Vallet F., 1991, «Études de dorures gallo-romaines et mérovingiennes ", Antiquités nationales, 22/23, Saint-Germain-en-Laye, p. 95-110.

Boucher S., 1976, Recherches sur les bronzes figurés de Gaule pré-romaine et romaine, Bibliothèque des Écoles françaises d'Athènes et de Rome, 228, Rome.

Bourgarit D., Mille B., 2003, "The elemental analysis of ancient copper-based artefacts by Inductively-CoupledPlasma Atomic-Emission-Spectrometry (ICP-AES): an optimized methodology reveals some secrets of the Vix Crater", Measurement Science and Technology, 14, 1538-1555.

Bourgeois B., 2013, "Greek art, French chemistry: an early encounter (late 18th-early 19 th centuries)", in M.-B. Failla, S. Adina Meyer, C. Piva, S. Ventra (a cura di), La cultura del restauro. Modelli di ricezione per la museologia e la storia dell'arte, Campisano Editore, Rome, p. 143-154.

Bourgeois B. (dir.), 2014, «Thérapéia. Polychromie et restauration de la sculpture dans l'Antiquité », Technè, 40.

Bret P., 2000, «Des essais de la monnaie à la recherche et à la certification des métaux : un laboratoire modèle au service de la guerre et de l'industrie (1775-1830) », Annales historiques de la Révolution française, 320, p. 1-12.

Cesareo R., Rizzutto M., Brunetti A., Rao D., 2009, "Metal location and thickness in a multilayered sheet by measuring $\mathrm{K} \alpha$ / K $\beta$, L $\alpha / \mathrm{L} \beta$ X-ray ratios”, Nuclear Instruments and Methods in Physic Research Section B: Beam interactions with Materials and Atoms, 267, p. 2890-2896.

Clarac F. comte de, 1841, Musée de sculpture antique et moderne [...], Imprimerie nationale, Paris, tome I, $841 \mathrm{p}$.

Darque-Ceretti E., Aucouturier M., 2012, Dorure : décor et sublimation de la matière, éditions Presses des mines, Paris.

Descamps-Lequime S., 2006, « La polychromie des bronzes grecs et romains ", dans Rouveret A., Dubel S. et Naas V., Couleurs et matières dans l'Antiquité. Textes, techniques et pratiques, ENS, Paris, p. 79-92.

Descamps-Lequime S., 2015, "The Color of Bronze Polychromy and the Aesthetics of Bronze Surfaces", in Exp. FlorenceLos Angeles-Washington, 2015, p. 151165.

Dorion-Peyronnet C., 2015, « L'Apollon de Lillebonne : histoire d'une découverte ", dans Exp. Rouen, 2015, p. 17-29.

Drayman-Weisser (ed.), 2000, Gilded Metals: History, Technology and Conservation, London.
Duval N., 1978, « L'Apollon de Lillebonne de l'abbé Cochet à nos jours. Tribulations et restaurations ", avec la collaboration de France-Lanord M. A., dans Centenaire de l'abbé Cochet-1975, Actes du colloque international d'archéologie, Rouen, p. 265-286.

Espérandieu E., Rolland H., Bronzes antiques de la Seine Maritime (XIII ${ }^{\mathrm{e}}$ supplément à Gallia), Paris.

Exp. Arles, 2009 : César : le Rhône pour mémoire, 2 ans de fouilles dans le fleuve à Arles [Exposition, Arles, Musée départemental Arles antique, 24 octobre 2009-19 septembre 2010, Long L., Picard P. (dir.)].

Exp. Évreux, 2013 : Grands dieux! Chefs-d'cuvre de la statuaire antique [Exposition, Evreux, Musée d'Evreux, 14 décembre 2013-23 mars 2014, F. Bridey (dir.)].

Exp. Florence-Los Angeles-Washington, 2015 : Power and Pathos. Bronze Sculpture of the Hellenistic World [Exposition, Florence, Palazzo Strozzi, 14 March-21 June 2015 ; Los Angeles, J. Paul Getty Museum, 28 July-1 November 2015 ; Washington D.C., National Gallery of Art, 6 December 2015-20 March 2016, Daehner J. M., Lapatin K. (eds.)], Giunti Editore, Fondazione Palazzo Strozzi, J. Paul Getty Trust.

Exp. Rouen, 2015 : Lillebonne-Juliobona. À la lumière des découvertes anciennes [Exposition, Rouen, Musée départemental des Antiquités, $1^{\mathrm{er}}$ avril$1^{\text {er }}$ juillet 2015, Dorion-Peyronnet C. (dir.)].

France-Lanord A., 1975 : « Musée du Louvre. Restauration de la statue de l'Apollon de Lillebonne ", note sommaire du 5 janvier 1975, tapuscrit conservé dans le dossier de l'œuvre au département des Antiquités grecques, étrusques et romaines, musée du Louvre.

Houtou Labillardière F.-J., 1824, « Sur la statue de Lillebonne », Annales de chimie et de physique, tome 26, p. 107-109.

Karidas A., 2007, "Application of a portable XRF spectrometer for the non-invasive analysis of museum metal artefacts", Annali di Chimica, 97, p. 419-432.

Lenormant F., 1875, « L'Apollon du VieilÉvreux ", Gazette archéologique, 1, p. 35-37.

Mille B., Nicot F., Robcis D., 2009, «Traitements de restauration et étude technique de la victoire en bronze d'or ", dans Exp. Arles 2009, p. 172-179.

Mille B., Rossetti L., Rolley C., avec la collaboration de Bourgarit D., Formigli E. et Pernot M., 2012, «Les deux statues d'enfant en bronze (Cap d'Agde) : étude iconographique et technique ", dans Denoyelle M., Descamps S., Mille B. et Verger S. (dir.), Actes du colloque Bronzes grecs et romains, recherches récentes, hommage à Claude Rolley, INHA, 16-17/06/2009, http://inha.revues.org/3949.

Oddy W., 1981, "Gilding through the ages", Gold Bulletin, 14/2, p. 75-79.

Oddy W. A., Cowell M. R., Craddock P. T., Hook D. R., "The Gilding of Bronze Sculpture in the Classical World", 1990, dans True M., Podany J., Small Bronze Sculpture from the Ancient World. Papers delivered at a Symposium Organized by the Department of Antiquities and Antiquities Conservation and Held at the J. Paul Getty Museum, March 16-19, 1989, the J. Paul Getty Museum, Malibu, California.

Oddy W., 1993, "Gilding of metals in the old world”, dans La Niece S., Craddock P., 1993, Metal plating and Patination, ed. Gutterworth, London, p. 171-181.

Rever, F. 1802, Voyage des Élèves du Pensionnat de l'École centrale de l'Eure, dans la partie occidentale du Département, pendant les vacances de l'an huit avec des Observations, des Notes et plusieurs Gravures relatives à l'Histoire Naturelle, l'Agriculture, les Arts, etc., Évreux : J. J. L. Ancelle, An X.

Rever F., 1821, Mémoire sur les ruines de Lillebonne, Évreux.

Rever F., 1824, Description de la statue fruste en bronze doré, trouvée à Lillebonne, arrondissement du Havre, Département de la Seine-Inférieure; suivie de l'Analyse du métal, avec le Dessin de la Statue, et les Tracés de quelques particularités relatives à la confection de cette Antique, Évreux, seconde édition revue par l'auteur.

Rogeret I., Carte archéologique de la Gaule. 76. La Seine-Maritime, Paris.

Roth-Zehner M., Le Martret A., Bailliot M., 2015, «Découverte de fragments de statue monumentale en bronze doré à Horbourg-Wihr (France, Alsace, HautRhin) ", dans Deschler-Erb E., Della Casa P. (dir.), New Research on Ancient Bronzes, Acta on the XVIII ${ }^{\text {th }}$ International Congress on Ancient Bronzes (Zurich, 3-7 September 2013), Zurich Studies in Archaeology 10, p. 117-120.

Schnitzler B., 1995, Bronzes antiques d'Alsace, Paris.

Shugar A., Mass J. (eds), 2012, Handled XRF for Art and Archaeology, Studies in Archaeological Sciences, Leuven.

Smith R., 1854, "Notes on Some of the Antiquities of France", Collectanea Antiqua III, Londres, pl. XXV.

Szewczyk M., 2013a, «Jupiter et Apollon. Considérations stylistiques et iconographiques », dans Exp. Évreux, 2013, p. 18-21.

Szewczyk M., 2013b, « Apollon au VieilÉvreux : de Lugus à saint Taurin, Dialogue d'histoire ancienne, vol. $39, \mathrm{n}^{\circ} 2$, p. 191-240.

Tailliez B., 1982, «Trente ans d'histoire de l'Apollon de Lillebonne », La Revue du Louvre et des Musées de France, avril $1982, n^{\circ} 2$, p. 81-88.

Vauquelin L.-N., 1824, « Analyse du métal de la statue trouvée à Lillebonne, près Caudebec, département de la SeineInférieure, dans un terrain appartenant à M. Holley ", Annales de chimie et de physique, tome 25, p. 395-401.

Zanker P., 1974, Klassizistische Statuen, Mayence. 